\title{
GluA4 enables associative memory formation by facilitating cerebellar expansion coding
}

\author{
Katarzyna Kita ${ }^{1,2}$, Catarina Albergaria ${ }^{3}$, Ana S. Machado ${ }^{3}$, Megan R. Carey ${ }^{3}$, \\ Martin Müller ${ }^{1,2} \&$ Igor Delvendahl ${ }^{1,2, *}$ \\ ${ }^{1}$ Department of Molecular Life Sciences, University of Zurich, Zurich \\ 2 Neuroscience Center Zurich, Zurich \\ ${ }^{3}$ Champalimaud Neuroscience Programme, Champalimaud Centre for the Unknown, Lisbon \\ * Correspondence to: igor.delvendahl@uzh.ch
}

\begin{abstract}
AMPA receptors (AMPARs) mediate excitatory neurotransmission in the CNS and their subunit composition determines synaptic efficacy. Whereas AMPAR subunits GluA1-GluA3 have been linked to particular forms of synaptic plasticity and learning, the functional role of GluA4 remains elusive. Here we used electrophysiological, computational and behavioral approaches to demonstrate a crucial function of GluA4 for synaptic excitation and associative memory formation in the cerebellum. Notably, GluA4-knockout mice had 80\% reduced mossy fiber to granule cell synaptic transmission. The fidelity of granule cell spike output was markedly decreased despite attenuated tonic inhibition and increased NMDA receptor-mediated transmission. Computational modeling revealed that GluA4 facilitates pattern separation that is important for associative learning. On a behavioral level, while locomotor coordination was generally spared, GluA4-knockout mice failed to form associative memories during delay eyeblink conditioning. These results demonstrate an essential role for GluA4-containing AMPARs in cerebellar information processing and associative learning.
\end{abstract}

\section{Introduction}

AMPA receptors (AMPARs) are essential for excitatory neurotransmission in the central nervous system (CNS). AMPARs are tetramers assembled from a combination of four subunits, GluA1-GluA4, that have distinct properties (Traynelis et al., 2010). AMPAR subunit expression shows strong regional variations (Geiger et al., 1995; Schwenk et al., 2014; Sjöstedt et al., 2020), suggesting specific functions in different types of neurons. Previous studies have revealed roles for AMPAR subunits GluA1-GluA3 in diverse forms of synaptic plasticity across the CNS (Citri et al., 2010; Gutierrez-Castellanos et al., 2017; Renner et al., 2017; Roth et al., 2020; Shi et al., 2001; Silva et al., 2019; Steinberg et al., 2006; Zamanillo, 1999), but much less is known about the function of the GluA4 subunit.

GluA4 confers rapid kinetics and large conductance to synaptic receptors (Mosbacher et al., 1994; Swanson et al., 1997). Yet, its expression is confined to few types of neurons in the adult brain (Keinänen et al., 1990; Monyer et al., 1991). Deletion of GluA4 impairs excitatory input to certain neurons in the auditory brainstem (Yang et al., 2011), the thalamus ( $\mathrm{Paz}$ et al., 2011; Seol and Kuner, 2015), and the hippocampus (Fuchs et al., 2007). In the cerebellum, granule cells (GCs)-the most abundant neurons in the mammalian brain-heavily express GluA4 (Cathala et al., 2005; Hollmann and Heinemann, 1994; Mosbacher et al., 1994; Schwenk et al., 2014), but the significance of GluA4 for GCs and for cerebellar circuit function remains unclear.

The cerebellum plays an important role for sensorimotor integration, motor coordination, motor learning and timing, as well as cognition (Diedrichsen et al., 2019; Raymond and Medina, 2018). The underlying computations are very fast, allowing millisecond precision in the calibration and timing of movement (Heck et al., 2013; Osborne et al., 2007). This astonishing speed is achieved by the cerebellar cortex with a highly conserved network structure: Sensory and motor information enter the cerebellar cortex via mossy fibers (MFs) that contact a large number of GCs. These small, numerous neurons greatly outnumber MFs, thus providing a large expansion of coding space (Albus, 1971; Marr, 1969). GCs relay information via their parallel fibers (PF) to Purkinje cells (PCs), the sole output neurons of the cerebellar cortex. Accumulating evidence suggests that the $\mathrm{MF} \rightarrow \mathrm{GC}$ synapse has evolved mechanisms allowing for transmission with exceptionally high rates and precision (Delvendahl and Hallermann, 2016; DiGregorio et al., 2002; Rancz et al., 2007). However, the molecular mechanisms underlying rapid signal integration in cerebellar GCs remain enigmatic.

Here we used electrophysiological recordings, computational modeling, and behavioral analyses to study cerebellar function in adult GluA4-knockout (GluA4-KO) mice. Deletion of GluA4 resulted in a selective impairment of $\mathrm{MF} \rightarrow \mathrm{GC}$ transmission. Despite compensatory changes in inhibition and NMDA receptor-mediated synaptic input, the pronounced decrease of AMPAR-mediated excitation caused a severe deficit in synaptic integration during highfrequency transmission. These synaptic changes impaired pattern separation and learning performance of a feedforward network model. On a behavioral level, GluA4$\mathrm{KO}$ mice displayed normal locomotor coordination, but a complete absence of eyeblink conditioning. Our findings demonstrate the importance of the GluA4 AMPAR subunit 
bioRxiv preprint doi: https://doi.org/10.1101/2020.12.04.412023; this version posted December 4,2020 . The copyright holder for this

preprint (which was not certified by peer review) is the author/funder, who has granted bioRxiv a license to display the preprint in perpetuity. It is made available under aCC-BY-NC-ND 4.0 International license.

for synaptic function and associative memory formation in the cerebellum.

\section{Results}

\section{Selective Impairment of Cerebellar MF $\rightarrow$ GC Synapses in GluA4-KO Mice.} with strongest detection in the cerebellum (Sjöstedt et al., 2020). Within the cerebellar cortex, this AMPAR subunit has been described in Bergmann glia (Saab et al., 2012) and in GCs (Mosbacher et al., 1994), where GluA4 is the major AMPAR subunit at MF $\rightarrow$ GC synapses (Delvendahl et
GluA4 shows a regional expression pattern in the CNS

al., 2019). To investigate the role of GluA4 for cerebellar function, we performed whole-cell patch-clamp recordings at excitatory synapses in slices of adult WT and GluA4-KO mice (Figure 1A). Recordings were made in the anterior vermis that mainly receives sensory MF input (lobules IIIIV; Giovannucci et al., 2017; Witter and De Zeeuw, 2015). GluA4-KO GCs displayed strongly diminished synaptic responses upon MF stimulation, with a $\sim 80 \%$ reduction in excitatory postsynaptic currents (EPSCs; $76.2 \pm 7.5 \mathrm{pA}$ vs. $13.4 \pm 1.1 \mathrm{pA} ; \mathrm{p}<0.0001$; Figure 1B-C; (Delvendahl et al., 2019)). Consistent with the fast kinetics of GluA4containing AMPARs (Mosbacher et al., 1994), EPSC decay kinetics were slower in GluA4-KO GCs $\quad(1.8 \pm 0.2 \mathrm{~ms} \quad$ vs.

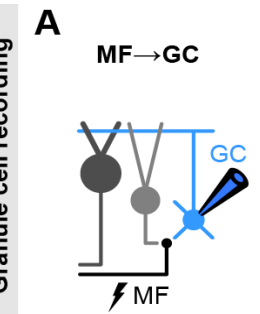

B
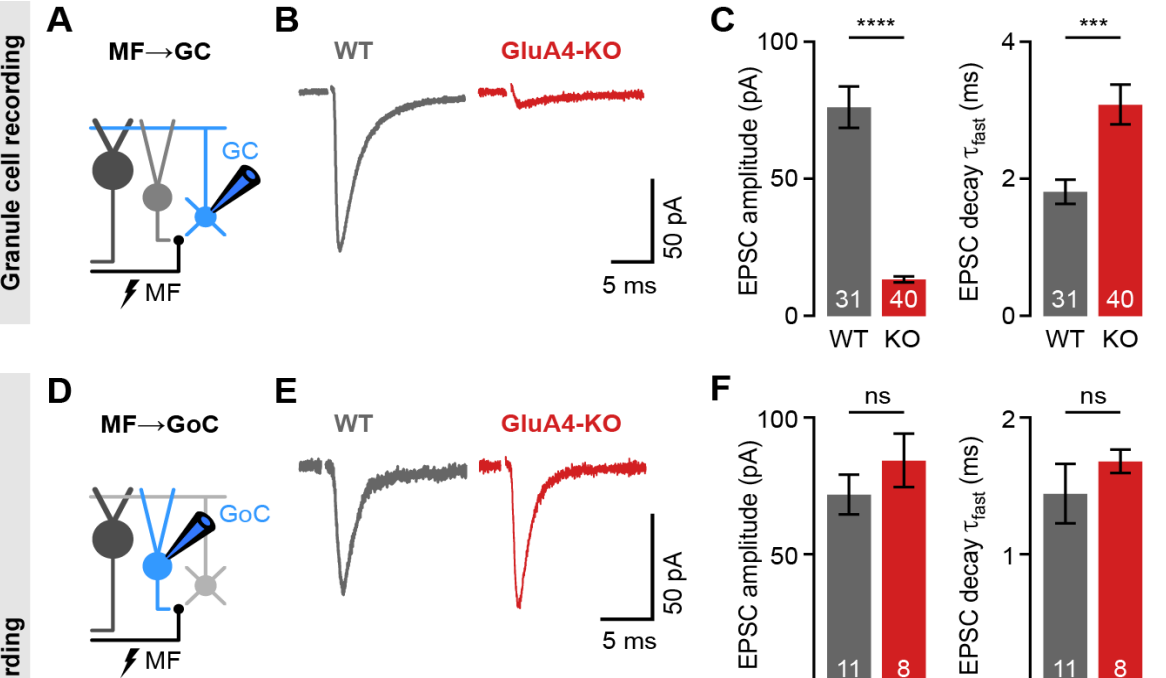

E

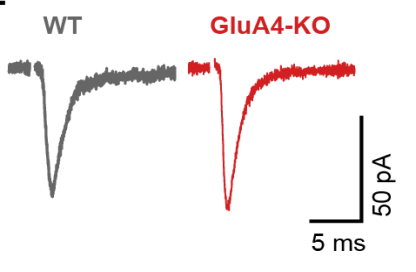

$\mathbf{F}$
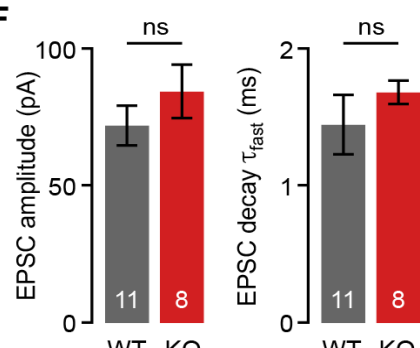

$\mathbf{G}$

H
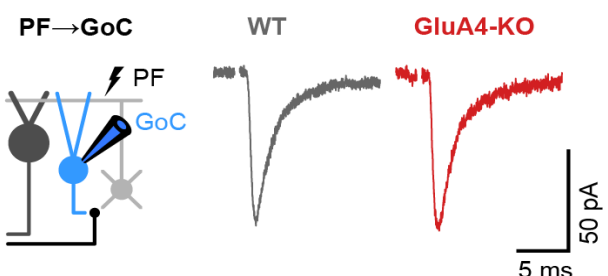

I
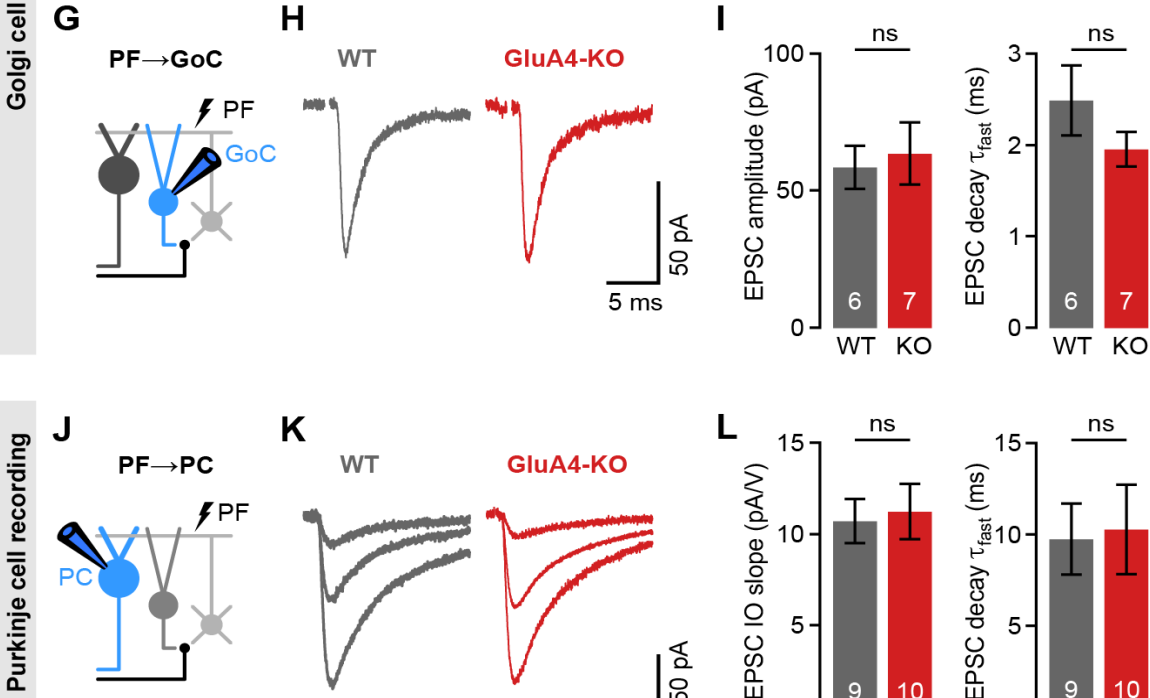

K

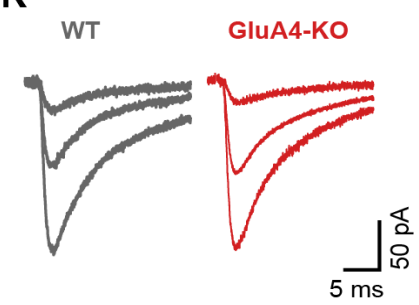

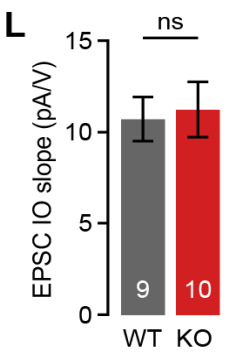

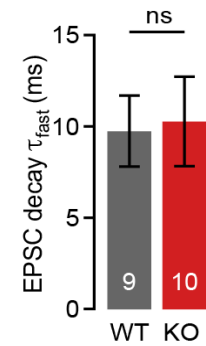

Figure 1. Selective Impairment of Cerebellar MF $\rightarrow$ GC Synapses in GluA4-KO Mice.

A. Recordings from MF $\rightarrow \mathrm{GC}$ connections. B. Example EPSCs recorded from WT and KO GCs by single MF stimulation. C. Average EPSC amplitude (left) and average EPSC fast decay time constant (right) for WT and KO MF $\rightarrow$ GC EPSCs. Data are redrawn from (Delvendahl et al., 2019). D. Recordings from $M F \rightarrow G o C$ connections. E. Example EPSCs upon MF stimulation recorded from WT and KO GoCs. F. Average EPSC amplitude (left) and average EPSC fast decay time constant (right) for WT and $\mathrm{KO} \mathrm{MF} \rightarrow \mathrm{GoC}$ EPSCs. G. Recordings from PF $\rightarrow$ GoC connections. H. Example EPSCs recorded from WT and KO GoCs upon PF stimulation. I. Average EPSC amplitude (left) and average EPSC fast decay time constant (right) for WT and KO PF $\rightarrow$ GoC EPSCs. J. Recordings from PF $\rightarrow P C$ connections. K. Example EPSCs recorded from WT and KO PCs by stimulating afferent PFs with increasing stimulation strength (displayed stimulation intensities: 7, 12 and $17 \mathrm{~V}$ ). L. Average linear slope of the input-output relationship (left) and average EPSC fast decay time constant (right) for WT and KO PF $\rightarrow$ PC. Data are means \pm SEM.
$3.1 \pm 0.3 \mathrm{~ms} ; \quad \mathrm{p}<0.001 ;$ Figure

1C). To probe the contribution of

GluA4 to excitatory synapse function along the MF pathway, we investigated MF synapses onto Golgi cells (GoCs), which provide feedforward and feedback inhibition to GCs (Duguid et al., 2015). At MF $\rightarrow$ GoC connections (Figure 1D), EPSC amplitudes and decay kinetics were comparable between WT and GluA4-KO (Figure 1E-F), suggesting that GluA4 does not contribute to $\mathrm{MF} \rightarrow \mathrm{GoC}$ transmission.

To probe if deletion of GluA4 affects the function of GC output synapses, we first investigated PF inputs to GoCs (PF $\rightarrow$ GoC synapses; Figure 1G), which were similar between WT and GluA4-KO mice (Figure $1 \mathrm{H}-$ I). Consistent with the similar EPSC amplitudes, spontaneous EPSCs and paired-pulse ratios were also not altered in GoCs of GluA4-KO mice (Figure S1A-D). We next studied $\mathrm{PF} \rightarrow \mathrm{PC}$ synapses (Figure 1J). Stimulation in the molecular layer with increasing intensity revealed similar $\mathrm{PF} \rightarrow \mathrm{PC}$ EPSCs in WT and GluA4-KO (Figure 1K). The slope of the input-output relationship was comparable between genotypes (Figure 1L; Figure S1G), which indicates normal recruitment of PFs. Likewise, EPSC decay kinetics (Figure 1L), as well as spontaneous EPSC amplitudes and paired-pulse ratios were similar in WT and KO 
PCs (Figure S1E-H), suggesting that GluA4 does not contribute directly to $\mathrm{PF} \rightarrow \mathrm{PC}$ synaptic transmission. Together, the recordings from $\mathrm{PF} \rightarrow \mathrm{GoC}$ and $\mathrm{PF} \rightarrow \mathrm{PC}$ synapses indicate that PF output is functionally intact in GluA4-KO mice. Our findings demonstrate that GluA4 is indispensable for fast neurotransmission at $\mathrm{MF} \rightarrow \mathrm{GC}$ synapses and suggest a GC-specific impairment of excitatory transmission at the cerebellar input layer in GluA4-KO mice.

al., 2009) and in vivo (Chadderton et al., 2004), we next probed $\mathrm{GC}$ firing in the presence of the $\mathrm{GABA}_{A}$ receptor $\left(G A B A_{A} R\right)$ blocker bicuculline. Interestingly, bicuculline increased spiking only in WT GCs, to a level that was comparable to GluA4-KO GCs (ANOVA: current $\times$ genotype $x$ drug: $F=5.34, p=0.02$; Figure $2 B-C$ ). Blocking inhibition increased the gain and reduced rheobase in WT (Figure 2D), consistent with enhanced intrinsic excitability. By contrast, bicuculline had no effect on gain and rheobase

current in GluA4-KO GCs (ANOVA gain: genotype $\times$ drug: $F=4.24$, $p=0.041$; rheobase: genotype $x$ drug: $F=2.00, p=0.16$; Figure 2D), indicating that tonic inhibition is reduced in these mice.

Persistent activation of $\delta$ and $\alpha 6$-containing extrasynaptic $\mathrm{GABA}_{\mathrm{A}} \mathrm{Rs}$ causes a tonic inhibitory conductance in cerebellar GCs (Brickley et al., 2001; Hamann et al., 2002; Stell et al., 2003) that also influences their excitability (Figure S2A-C; (Mitchell and Silver, 2003; Rudolph et al., 2020)). We directly investigated tonic inhibition by isolating inhibitory conductance in WT and GluA4-KO GCs and bath-applying bicuculline. Indeed, GluA4-KO GCs had a smaller root-mean-square noise of the baseline holding current and smaller bicuculline-sensitive
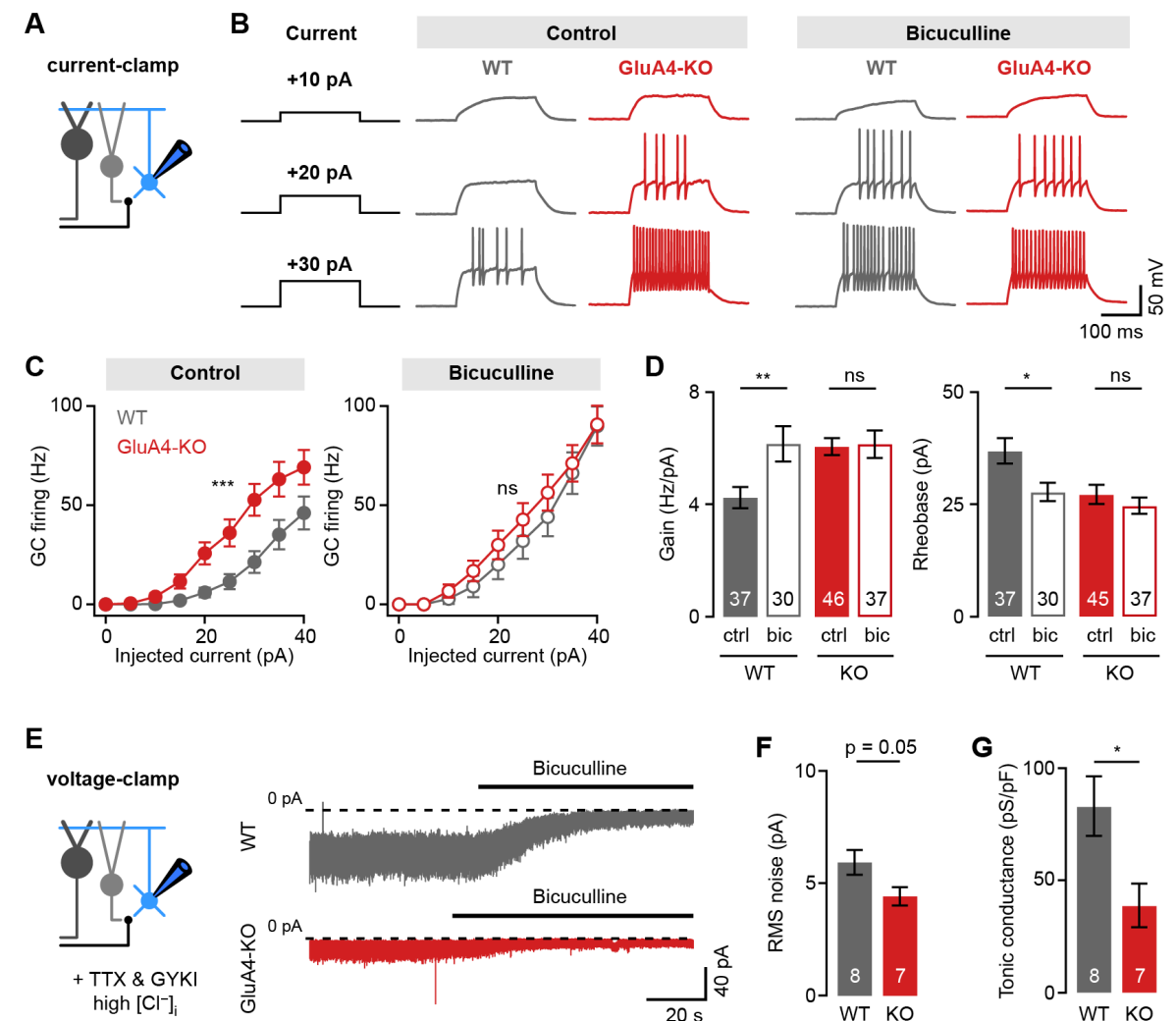

Figure 2. Reduced Tonic Inhibition Enhances GC Intrinsic Excitability.
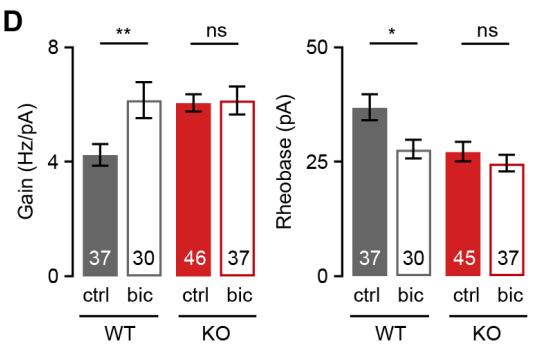

A. Current-clamp recordings from GCs. B. Example responses to somatic current injection of indicated amplitude for control and in the presence of $10 \mu \mathrm{M}$ bicuculline. C. Average GC firing frequency plotted vs. injected current for WT and $\mathrm{KO}$ in control and bicuculline. D. Average gain and rheobase, calculated from the frequency-current curves in (C). In the presence of bicuculline, KO GCs had gain and rheobase values comparable to WT. E. Recording of tonic holding current. GABAergic currents were isolated using $20 \mu \mathrm{M}$ GYKI-53655 and high intracellular $\mathrm{Cl}^{-}$. Right: Example recordings of bicuculline wash-in $(10 \mu \mathrm{M})$ for WT and KO GCs. F. Left: Average root-mean-square noise before washin of bicuculline (left) and average tonic conductance density (right) for WT and GluA4-KO. Tonic conductance was calculated from the bicuculline-sensitive current. Data are means \pm SEM. conductance compared with WT (WT: $\quad 83.0 \pm 13.3 \mathrm{pS} / \mathrm{pF}, \quad \mathrm{KO}$ : $39.0 \pm 9.6 \mathrm{pS} / \mathrm{pF}, \quad \mathrm{p}=0.022$; Figure 2E-F). These findings demonstrate that tonic $\mathrm{GABA}_{A} \mathrm{R}$ mediated inhibition is reduced in GluA4-KO GCs, in line with the marginal effect of bicuculline on

\section{Reduced Tonic Inhibition Enhances GC Intrinsic Excitability.}

Loss of GluA4 severely compromises synaptic excitation of cerebellar GCs. Changes in both excitatory and inhibitory synaptic input may influence a neuron's intrinsic excitability (Aizenman and Linden, 2000; Desai et al., 1999; Karmarkar and Buonomano, 2006). To investigate if GluA4-KO alters the intrinsic excitability of GCs, we quantified GC spiking upon increasing tonic current injections (Figure 2A). Under control conditions, GluA4-KO GCs showed higher firing frequencies than WT, reflecting increased excitability (Figure $2 \mathrm{~B}-\mathrm{C}$ ). Because GoC-mediated tonic inhibition influences the intrinsic excitability of GCs both in slices (Brickley et al., 2001; Hamann et al., 2002; Mitchell and Silver, 2003; Rothman et intrinsic excitability of GluA4-KO GCs. By contrast, the amplitudes and frequencies of spontaneous inhibitory postsynaptic currents (sIPSCs) were not different between WT and GluA4-KO GCs (Figure S2E), indicating that phasic inhibition is not altered. To verify the specificity of our findings, we also studied excitability in GCs of heterozygous GluA4 mice (GluA4-HET), which have reduced GluA4 levels (Figure S5A) but no change in $\mathrm{MF} \rightarrow \mathrm{GC}$ EPSC amplitudes (Figure S5E-F; (Delvendahl et al., 2019)). We did not observe indications of altered inhibition in GluA4-HET GCs (Figure S5B-D). Thus, impaired excitatory synaptic input to GCs is accompanied by a modulation of tonic inhibition, resulting in increased intrinsic excitability. 
A

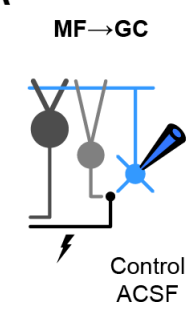

B

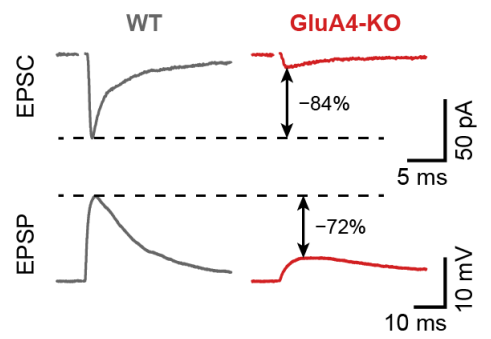

C

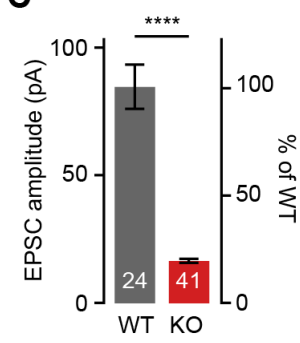

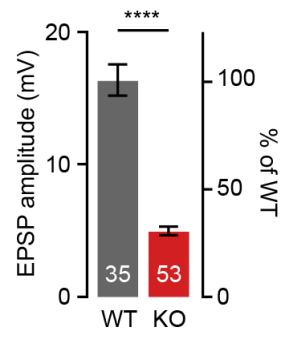
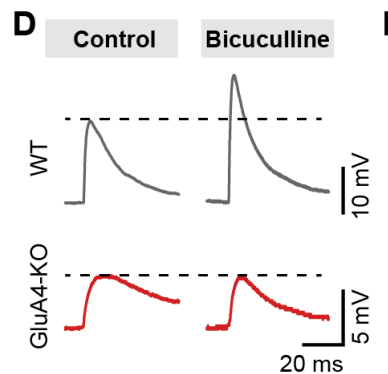

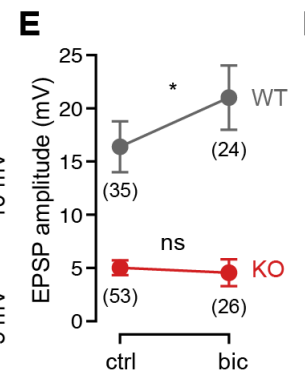

$\mathbf{F}$
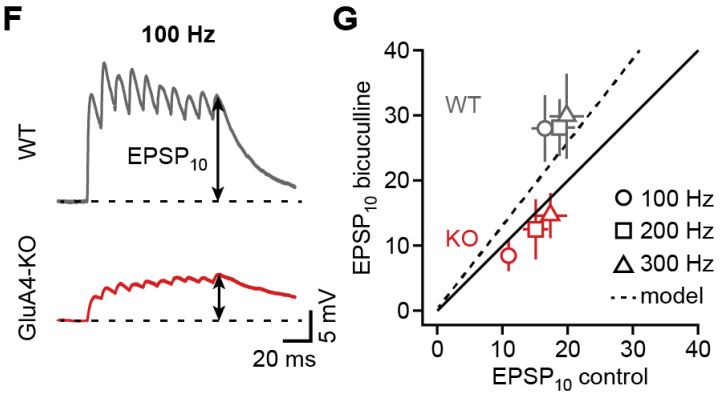

Figure 3. Shunting Inhibition Controls EPSP Size in Cerebellar GCs.

A. Recordings from MF $\rightarrow G C$ connections in voltage- or current-clamp with blocker-free ACSF ('control'). B. Example EPSC and EPSP recordings from the same cell in WT and KO. Arrows indicate reduction compared to WT. C. Average EPSC and EPSP amplitudes for WT and KO. Note the difference in relative reduction of EPSPs compared to EPSCs. D. Example EPSP recordings for control and in the presence of $10 \mu \mathrm{M}$ bicuculline. E. Average EPSP amplitude for WT and KO. Error bars represent $95 \% \mathrm{Cl}$. F. Example MF $\rightarrow$ GC EPSP trains $(10$ stimuli, $100 \mathrm{~Hz}$ ). G. Average EPSP 10 in the presence of bicuculline vs. control for the indicated stimulation frequencies. Black line represents unity and dashed line the prediction of an integrate-and-fire model with or without tonic inhibition (cf. Figure S2). Data in (C) and (G) are means \pm SEM.

\section{Shunting Inhibition Controls EPSP Size in Cerebellar GCs.}

Tonic GABAAR activation leads to shunting inhibition of GCs that may affect their responses to excitatory input (Brickley et al., 2001; Mitchell and Silver, 2003). The reduction of tonic inhibition in GluA4-KO GCs is therefore expected to impact excitatory postsynaptic potentials (EPSPs). To assess the impact of shunting inhibition in WT and GluA4-KO GCs, we recorded MF $\rightarrow$ GC EPSCs and EPSPs in blocker-free solution ('control', Figure 3A). As expected, EPSC amplitudes were strongly diminished in GluA4-KO GCs, as were EPSP amplitudes (Figure 3B-C). Interestingly, the amplitude reduction relative to WT was smaller for EPSPs than for EPSCs $(69.4 \pm 2.1 \%$ vs. $80.6 \pm 1 \%, p<0.001$; Figure $3 C$ ). To test if reduced tonic inhibition underlies this effect, we recorded EPSPs in the presence of bicuculline, which increased EPSP amplitudes in WT, but not in GluA4-KO GCs (ANOVA drug $\times$ genotype: $F=8.00, p=0.005$; Figure $3 D-E)$. We also observed that bicuculline enhanced EPSP amplitudes during highfrequency train stimulation in WT, but not in GluA4-KO GCs (ANOVA drug $\times$ genotype: $F=46.88, p<0.001$; Figure 3F$G)$. In contrast, bicuculline had no differential effect on EPSC amplitudes (ANOVA drug $\times$ genotype: $F=0.35$, $p=0.56$; Figure $S 2 F-G$ ). These results demonstrate that shunting inhibition reduces postsynaptic depolarization in response to excitatory input in WT, but not in GluA4-KO GCs. We conclude that the reduced tonic inhibition upon loss of the major AMPAR subunit at MF $\rightarrow$ GC synapses augments synaptic depolarization in GluA4-KO GCs.

Deletion of GluA4 Impairs GC Spike Fidelity and Precision during High-Frequency Transmission.
How do the changes in excitatory input and intrinsic excitability influence GC firing in response to MF input? To address this question, we performed current-clamp recordings from GCs in combination with high-frequency $\mathrm{MF} \rightarrow \mathrm{GC}$ stimulation (Figure $4 \mathrm{~A}$ ) at a membrane potential of $-70 \mathrm{mV}$, corresponding to the average resting membrane potential of GCs in vivo (Chadderton et al., 2004; Powell et al., 2015). WT GCs showed increasing firing frequencies upon $100-300 \mathrm{~Hz}$ stimulation (Figure $4 \mathrm{~B}-\mathrm{C}$ ). We also observed MF-stimulation-evoked spikes in GluA4KO GCs, albeit at reduced frequency (ANOVA genotype: $F=23.48 ; p<0.0001$; Figure $4 B$ ) and in fewer cells (Figure $4 C)$. The GC spikes elicited by high-frequency $M F \rightarrow G C$ stimulation were not only reduced in number, but also occurred with a longer delay compared to WT (ANOVA genotype: $F=26.26 ; p<0.0001$; Figure 4D-E). Hence, GluA4-KO GCs need to summate more EPSPs to reach spike threshold (Figure S3D), leading to reduced reliability and impaired temporal precision of GC spikes. Spike output was not altered in GCs of GluA4-HET mice (Figure $\mathrm{S} 5 \mathrm{H}-\mathrm{I})$. Together, these data show a strong reduction of spiking frequency and temporal precision in GluA4-KO GCs upon high-frequency synaptic input, and establish that GluA4-containing AMPARs control the timing and reliability of EPSP-spike coupling at the cerebellar input layer.

\section{NMDARs and Reduced Inhibition Support Spiking in GluA4-KO GCs.}

Given the strong reduction of MF $\rightarrow$ GC EPSCs (Figure 1), the occurrence of spikes upon repetitive stimulation in more than $30 \%$ of GluA4-KO GCs is surprising. We previously provided evidence for enhanced presynaptic 
A
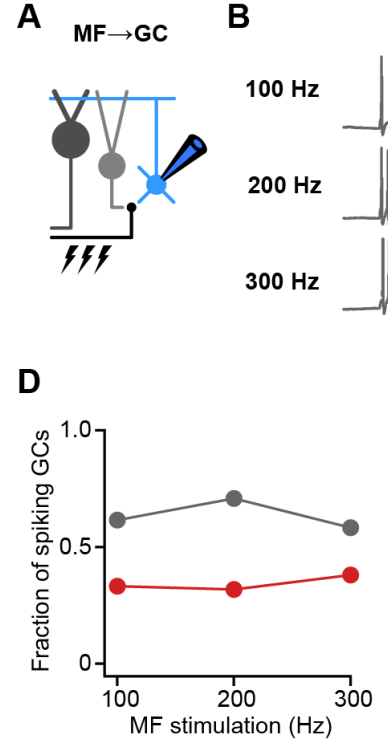

B

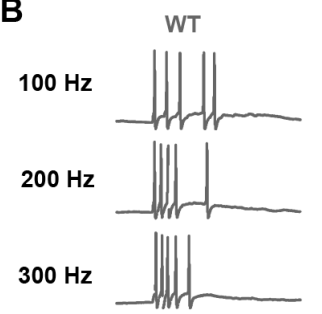

E

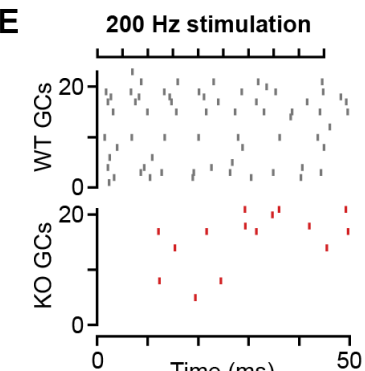

C

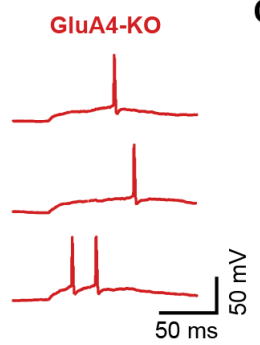

$\mathbf{F}$

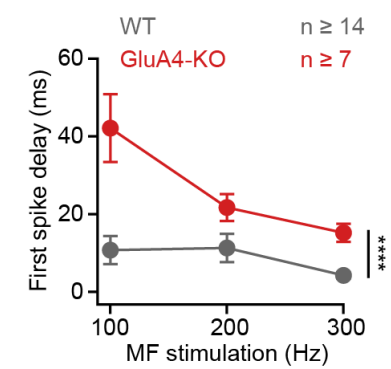

Figure 4. Deletion of GluA4 Impairs GC Spike Fidelity and Precision during High-Frequency Transmission.

A. Recordings from MF $\rightarrow \mathrm{GC}$ connections in current-clamp. B. Example voltage recordings from a WT and GluA4-KO GC upon MF stimulation with 10 stimuli at increasing frequencies (indicated). C. Average GC firing frequency upon MF stimulation with $100-300 \mathrm{~Hz}$ frequency. GCs were held at $-70 \mathrm{mV}$. D. Fraction of GCs showing spikes upon MF stimulation with $100-300 \mathrm{~Hz}$ frequency. E. Spike raster plots for $200 \mathrm{~Hz}$ MF stimulation in WT and GluA4-KO. F. Average first spike delay calculated from stimulation onset plotted versus MF stimulation frequency. Data are means \pm SEM.

glutamate release from GluA4-KO MF boutons (Delvendahl et al., 2019), which might increase the non-AMPAR component of $\mathrm{MF} \rightarrow \mathrm{GC}$ transmission. Indeed, isolated NMDA receptor (NMDAR)-mediated MF $\rightarrow$ GC EPSCs were larger in GluA4-KO than in WT GCs $(27.5 \pm 2.1 \mathrm{pA}$ vs. $37.2 \pm 2.1 \mathrm{pA} ; \mathrm{p}=0.0029$; Figure $5 \mathrm{~A}-\mathrm{B})$. Because NMDAR activation can significantly contribute to GC excitation during repetitive input (D'Angelo et al., 1995), we recorded isolated AMPAR- and NMDAR-EPSCs upon high-frequency GNMDA, respectively; Figure 5E) to model synaptic input of an integrate-and-fire neuron (Rothman et al., 2009). Simulations reproduced the experimentally observed GC firing and first spike delay well for WT and GluA4-KO upon fixed frequency MF input with a binomial short-term plasticity model (Rothman and Silver, 2014) (Figure S3BC). To probe the impact of tonic inhibitory conductance and GNMDA enhancement, we simulated four independent,
A

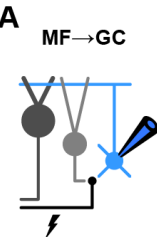

$\mathrm{Mg}^{2+}$ free ACSF + NBQX
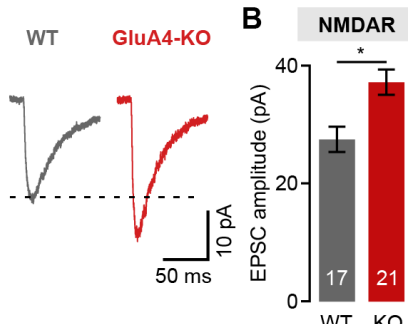

C

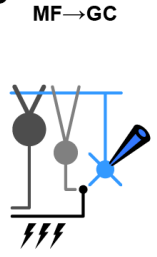

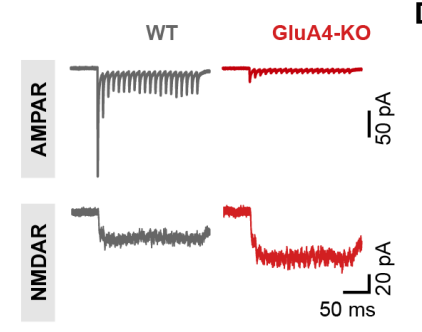

D AMPAR

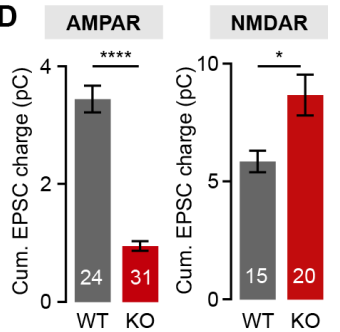

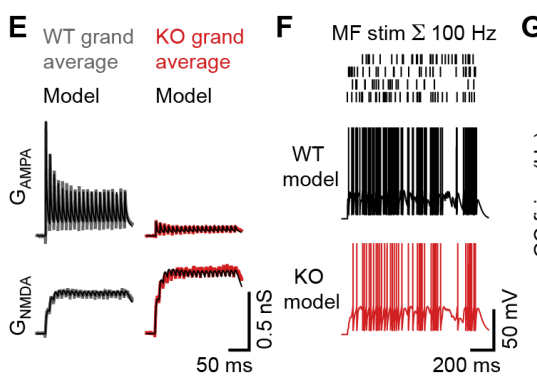
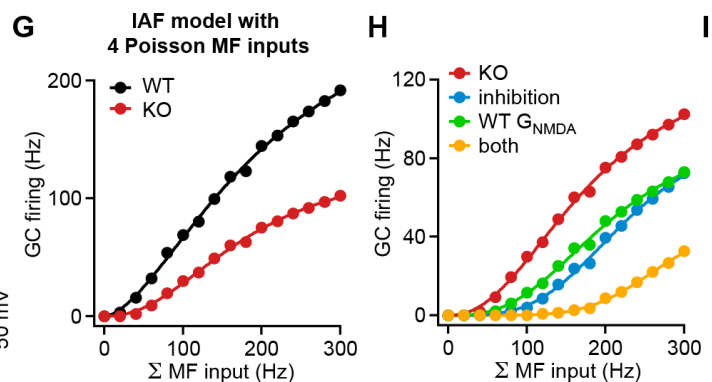

I

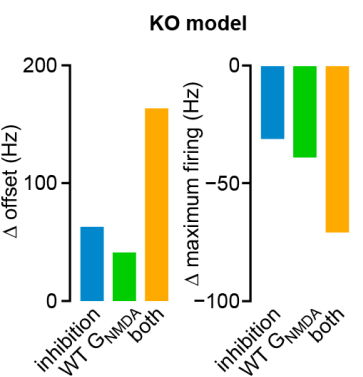

Figure 5. NMDARs and Reduced Inhibition Support Spiking in GluA4-KO GCs.

A. Left: Recordings of isolated NMDAR-EPSCs at MF $\rightarrow$ GC connections. Right: Example NMDAR-mediated MF $\rightarrow$ GC EPSCs. B. Average NMDAR-EPSC amplitude (right) for WT and GluA4-KO GCs. C. Top: Example AMPAR-EPSC 100-Hz train recordings (20 stimuli) for WT and KO. Bottom: Example NMDAR-mediated 100-Hz trains. D. Average cumulative charge for AMPAR-mediated (left) and NMDAR-mediated EPSCs (right) for both genotypes. E. Grand average of AMPAR- and NMDAR-conductance trains (20 stimuli, $100 \mathrm{~Hz}$ ) recorded from WT (left) and GluA4-KO GCs (right). Data are overlaid with synaptic conductance models for WT and GluA4-KO. F. Top: Four independent Poisson-distributed MF spike trains with sum of $100 \mathrm{~Hz}$ (top) and simulation results for WT (black) and KO (red) (bottom). G. Average WT and KO model firing frequency versus MF stimulation frequency. Lines are fits with a Hill equation. H. Results for KO model compared with simulations of KO GCs with tonic inhibition (blue), with WT $\mathrm{G}_{\mathrm{NMDA}}$ (green) or both (yellow). I. Reduced inhibition and enhanced GNMDA increase the maximum firing frequency (left) and reduce the offset (right) of the KO model GC output. Data are means \pm SEM. 
bioRxiv preprint doi: https://doi.org/10.1101/2020.12.04.412023; this version posted December 4 , 2020. The copyright holder for this

preprint (which was not certified by peer review) is the author/funder, who has granted bioRxiv a license to display the preprint in perpetuity. It is made available under aCC-BY-NC-ND 4.0 International license.

Poisson-distributed MF inputs (Figure 5F) covering the wide range of $M F$ frequencies observed in vivo (Arenz et al., 2008; van Kan et al., 1993). The model with GluA4-KO GAMPA and $\mathrm{G}_{\text {NMDA }}$ and reduced tonic conductance displayed lower firing frequencies over the entire range of MF inputs (Figure 5G; Figure S4A). Compared with WT, the KO model had a $\sim 44 \%$ reduced maximum firing frequency $(186.1 \mathrm{~Hz}$ vs. $336.4 \mathrm{~Hz}$ ) and a $17 \mathrm{~Hz}$ increased offset, similar to the experimental findings (Figure 4C). As in the current-clamp recordings (Figure $4 \mathrm{~F}$ ), spikes occurred with a longer delay in the KO GC model over all simulated MF frequencies (Figure S4D).

To understand the individual contributions of reduced tonic inhibition and enhanced G GMDA to spiking in KO GCs, we either added an inhibitory conductance of $0.16 \mathrm{nS}$ (calculated from Figure 2F) or used the WT GNMDA in the KO model. In both scenarios, firing frequencies were reduced in relation to the $\mathrm{KO}$ model, with each mechanism contributing $\sim 20 \%$ to the maximum spiking frequency of the $\mathrm{KO}$ model (Figure $5 \mathrm{H}-\mathrm{I}$, Figure S4A-B). Combining inhibition and WT GNMDA reduced the firing rate by $\sim 40 \%$ (Figure $5 \mathrm{H}-\mathrm{I}$ ), implying a synergistic, linear interaction of the two.

We next asked how the experimentally observed changes in GluA4-KO GCs influence synaptic information transfer. Analysis of pre- and postsynaptic spike trains revealed that the strongly reduced GAMPA of the KO model compromises the synchrony of MF and GC spikes ( 2-fold reduction in SPIKE synchronization; Figure S4F; (Mulansky et al., 2015)), and that the reduced tonic inhibition and GNMDA enhancement in GluA4-KO facilitate $\mathrm{MF} \rightarrow \mathrm{GC}$ synaptic information transfer at low input frequencies, albeit at a reduced level (Figure 5G-H, Figure S4E-F). Thus, although $\mathrm{MF} \rightarrow \mathrm{GC}$ AMPAR-EPSCs are strongly reduced, synaptic and non-synaptic mechanisms cooperatively counteract the impaired spiking output of GluA4-KO GCs. However, both mechanisms are insufficient to compensate for the pronounced impairment of synaptic excitation caused by the deletion of GluA4.

\section{Impaired Pattern Separation in a Feedforward Model of the Cerebellar Input Layer.}

The GC layer is ideally suited for sparsening and expanding of MF inputs (Albus, 1971; Marr, 1969). This transformation of neural coding is thought to enable effective pattern separation and facilitate associative learning in downstream circuits (Cayco-Gajic and Silver, 2019). To address how the reduced synaptic input in GluA4-KO GCs affects information processing in the GC layer, we employed computational modeling of a feedforward $\mathrm{MF} \rightarrow \mathrm{GC}$ network with constrained connectivity and biophysical properties (Figure 6A-B; (Cayco-Gajic et al., 2017)). We systematically varied the fraction of active MFs and their spatial correlation, and analyzed how the GC network transforms MF activity patterns (Cayco-Gajic et al., 2017). To assess the impact of reduced synaptic excitation, we scaled model synaptic
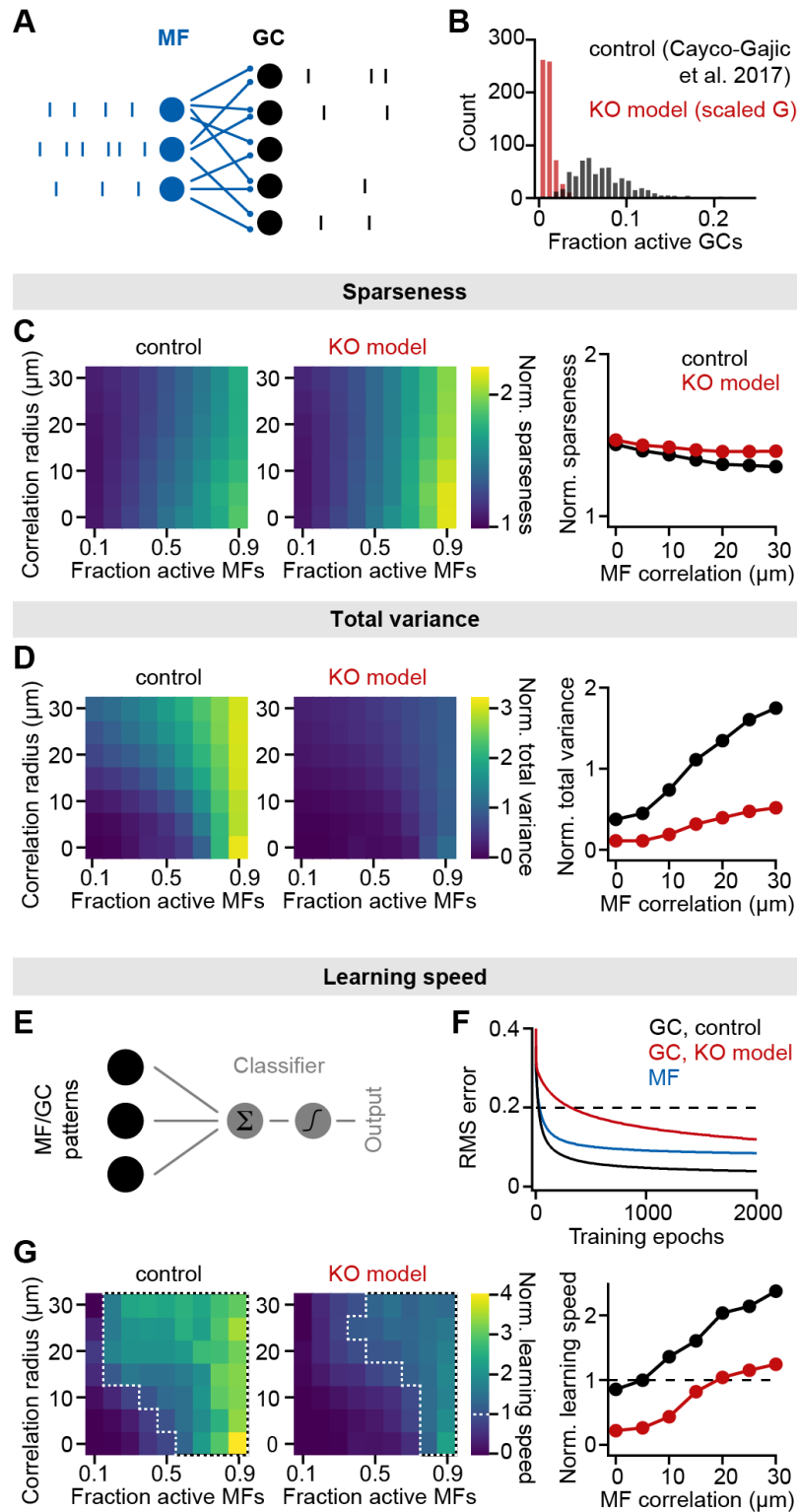

Figure 6. Impaired Pattern Separation in a Feedforward Model of the Cerebellar Input Layer.

A. Schematic of the feedforward network model. The model comprises $187 \mathrm{MFs}$ that are connected to $487 \mathrm{GCs}$ with 4 synapses per GC. The network is presented with different MF activity patterns, which produce GC spike patterns. B. Histogram of active GCs. Scaling synaptic and tonic conductances according to GluA4-KO data ("KO model", red) leads to strong reduction of active GCs compared to the model from Cayco-Gajic et al. (2017) ("control", black). C. Left: Normalized population sparseness (normalized to MF sparseness) plotted for different MF correlation radii and fraction of active MFs of both models. Right: Median normalized sparseness versus MF correlation radius. D. Same as C, but for total variance. E. Schematic of learning. MF or GC activity patterns are used to train a perceptron decoder to classify these patterns into 10 random classes. F. Root-mean-square (RMS) error of the perceptron classification for MF input patterns (blue), GC input patterns (black) or GC patterns with scaled conductance (red). Dashed line indicates cutoff for learning speed quantification. G. Left: Normalized learning speed (normalized to learning using MF patterns) plotted for different MF correlation radii and fraction of active MFs of both models. White dashed borders indicate areas of faster learning with GC activity patterns than with MF patterns. Right: Median normalized learning speed versus MF correlation radius. Dashed line indicates faster $\mathrm{GC}$ learning.

conductances and tonic inhibition according to our GluA4KO data (see Methods). Both control and KO models showed a similar increase in spatial sparseness of GC activity patterns compared to MF activity patterns (Figure 
A

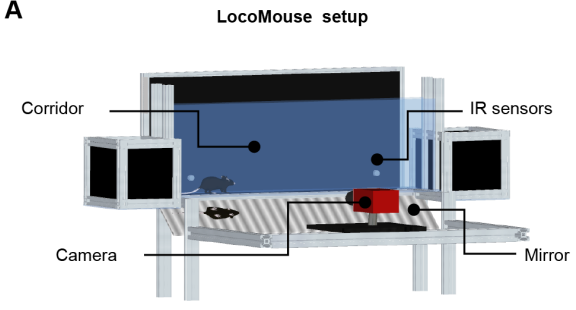

B
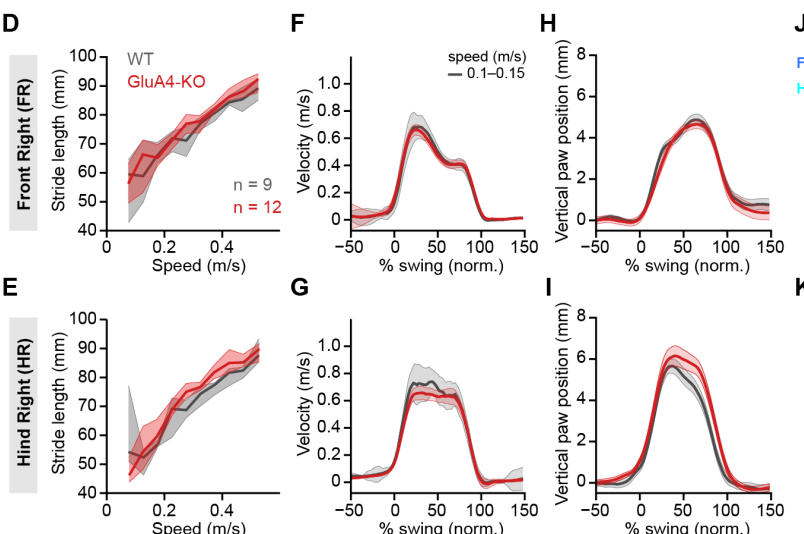

K

Figure 7. Normal Locomotor Coordination of GluA4-KO Mice During Overground Walking

A. LocoMouse apparatus. B. Continuous tracks (in $x, y, z$ ) for nose, paws and tail segments obtained from LocoMouse tracking (Machado et al., 2015) are plotted on top of the frame. C. Individual strides were divided into swing and stance phases for further analysis. D-E. Stride length of FR paw (D) and HR paw (E) vs. walking speed for GluA4-KO mice (red) and WT animals (gray). For each parameter, the thin lines with shadows represent median values \pm 25 th, 75th percentiles. F-G. Average instantaneous forward $(x)$ velocity of FR paw $(F)$ and HR paw (G) during swing phase. $\mathbf{H}-\mathbf{I}$. Average vertical (z) position of FR paw $(\mathbf{H})$ and HR paw (I) relative to ground during swing. The shaded area indicates SEM across mice. J-K. Polar plots indicating the phase of the step cycle in which each limb enters stance, aligned to stance onset of FR paw (red circle). Distance from the origin represents walking speed. J. WT mice; K. GluA4-KO mice. Circles show average values for each animal.

$6 \mathrm{C})$, indicating that deletion of GluA4 did not affect population sparsening. In addition to sparsening, an important characteristic of the GC layer is an expansion of coding space, which promotes pattern separation by increasing the distance between activity patterns. To investigate expansion recoding, we analyzed the size of the distribution of activity patterns by calculating the total variance of GC population activity, normalized to the total variance of the MF population activity (Cayco-Gajic et al., 2017). The normalized total variance-reflecting the expansion of coding space-was strongly reduced in the GluA4-KO model (Figure 6D), suggesting that the increased spiking threshold of GluA4-KO GCs leads to a strong reduction of coding space at the cerebellar input layer.

To address the consequences of alterations in sparsening and expansion recoding for learning performance, we trained a perceptron to classify either MF or GC activity patterns into ten random classes (Figure 6E), and analyzed learning speed using $\mathrm{GC}$ patterns normalized to MF patterns (Cayco-Gajic et al., 2017) (Figure 6F). Learning speed was reduced over the full range of model parameters when using the GluA4-KO synaptic conductances (Figure 6G). Notably, GC activity accelerated learning only at high correlation levels and active fraction of MFs, in contrast to the previously published model (Cayco-Gajic et al., 2017) (white borders in Figure 6G). This result is consistent with our current-clamp experiments, where higher MF stimulation frequencies elicited GC spikes in GluA4-KO mice (Figures $4 \mathrm{C}$ and S3A). Moreover,

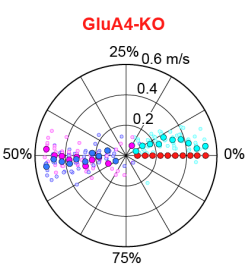

the total variance (Figure 6D)-but not population sparseness-closely predicted the learning speed, suggesting that the strong decrease of $\mathrm{MF} \rightarrow \mathrm{GC}$ transmission impairs pattern separation by reducing the coding space. Thus, GluA4 speeds learning by improving pattern separation performance in a feedforward $\mathrm{MF} \rightarrow \mathrm{GC}$ network model without affecting GC population sparseness.

\section{Normal Locomotor Coordination of GluA4-KO Mice during Overground Walking.}

We next investigated the functional consequences of the extreme reduction in $\mathrm{MF} \rightarrow \mathrm{GC}$ transmission in GluA4-KO mice for cerebellum-dependent behaviors. Cerebellar dysfunction often leads to impairments in motor coordination, including gait ataxia during walking (Morton and Bastian, 2007). We analyzed the locomotor behavior of GluA4-KO mice using the LocoMouse system (Machado et al., 2015) (Figure 7A). GluA4-KO mice walked similarly to size-matched WT littermates without obvious gait ataxia (Supplementary Video 1). Quantitative analysis of locomotor coordination (Machado et al., 2015, 2020) (Figure 7B-C) revealed that their locomotor behavior was largely intact. GluA4-KO mice tended to walk more slowly than controls, but across walking speeds, stride lengths were comparable in mice of both genotypes (Figure 7D-E, Figure S6A-B). Paw trajectories measured by continuous forward velocity were also intact (Figure 7F$\mathrm{G}$, Figure $\mathrm{S} 6 \mathrm{E}-\mathrm{F})$. Vertical paw motion was also largely normal (Figure $7 \mathrm{H}-\mathrm{I}$ ), although there was a small degree of hyperflexion across walking speeds (Figure S6F-G). We also explored interlimb coordination, which is often disrupted by cerebellar damage (Machado et al., 2015, 2020; Morton and Bastian, 2007), during normal walking. We did not observe any differences between WT and GluA4-KO mice, with both genotypes displaying a symmetrical trot pattern across walking speeds (Figure $7 \mathrm{~J}-\mathrm{K}$ ). Exhaustive analysis of the movements of individual limbs, interlimb and body coordination, and comparison with other mouse models of cerebellar ataxia confirmed the grossly normal locomotor phenotype of GluA4-KO mice (Figures S6-7). Moreover, GluA4-HET mice showed normal overall locomotion performance (Figure S8A-H). Thus, locomotor coordination was spared in GluA4-KO mice despite the pronounced impairment of synaptic excitation at the cerebellar input layer. 
A
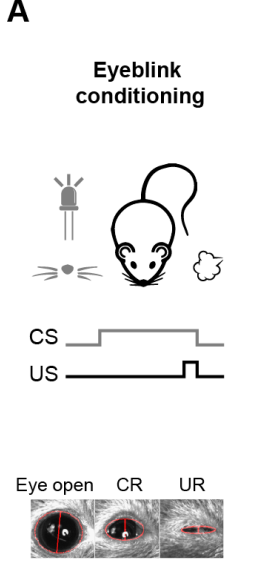

B
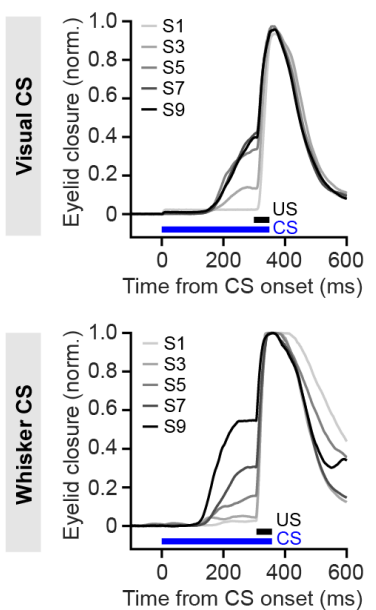

C
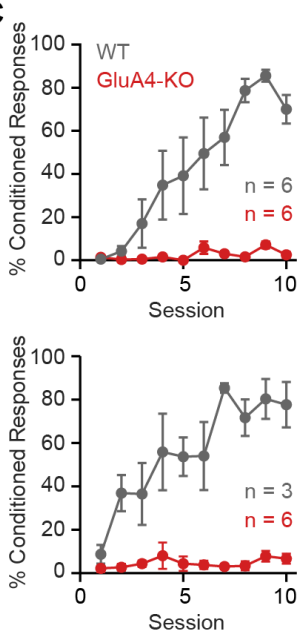

D
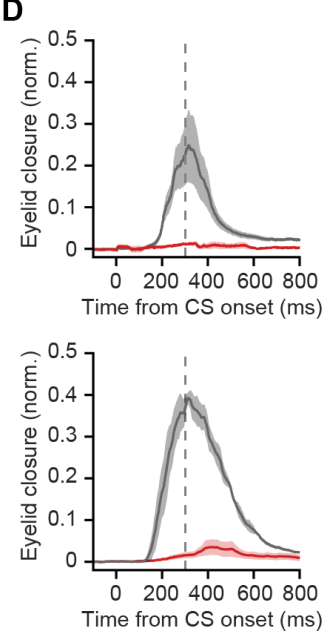

E
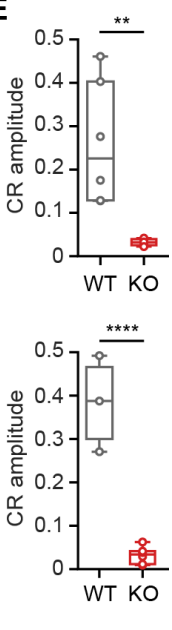

Figure 8. GluA4 is Required for Cerebellum-Dependent Associative Memory Formation.

A. Top: Schematic of the eyeblink conditioning paradigm, where a CS (a white LED or a whisker vibratory stimulus) is repeatedly paired and co-terminates with an eyeblinkeliciting US (a puff of air delivered to the eye). Bottom: Example video frames (acquired at $900 \mathrm{fps}$ under infrared light) illustrate automated extraction of eyelid movement amplitude. B. Average eyelid closure across 9 learning sessions (S1-S9) of two representative WT animals, using a visual CS (top) or a whisker CS (bottom). Each trace represents the average of 100 paired trials from a single session. C. Average \%CR learning curves of WT (gray) and GluA4-KO (red) mice trained with either a visual CS (top) or a whisker CS (bottom). Error bars indicate SEM. D. Average eyelid traces of CS-only trials from the last training session of WT and GluA4-KO animals, trained with either a visual CS (top) or a whisker CS (bottom). Shadows indicate SEM. Vertical dashed line represents the time that the US would have been expected on CS+US trials. E. Average CR amplitudes from the last training session of WT and GluA4-KO animals, trained with either a visual CS (top) or a whisker CS (bottom). Box indicates median and 25th-75th percentiles; whiskers extend to the most extreme data points.

\section{GluA4 is Required for Cerebellum-Dependent Associative Memory Formation.}

Influential theories of cerebellar function have proposed that pattern separation at the input layer facilitates learning within the cerebellar cortex (Albus, 1971; Cayco-Gajic and Silver, 2019; Marr, 1969). Thus, while the strong reduction of GC synaptic excitation did not impair locomotor coordination of GluA4-KO mice, it might specifically affect cerebellum-dependent learning. We therefore examined delay eyeblink conditioning in GluA4KO mice, a cerebellum-dependent form of associative learning that involves MF inputs conveying the conditioned stimulus (CS) (Albergaria et al., 2018; De Zeeuw and Yeo, 2005; Mauk, 1997). A conditioning light pulse was repeatedly paired with an unconditioned air-puff stimulus (US) to the eye as described previously (Albergaria et al., 2018) (Figure 8A). Over training sessions, WT animals acquired a conditioned eyelid closure that preceded the US (Figure 8B). WT mice displayed a continuous increase in the percentage of trials with a conditioned response (CR; Figure $8 \mathrm{C}$ ), demonstrating associative memory formation. Strikingly, GluA4-KO mice failed to develop CRs over the ten training sessions (Figure $8 \mathrm{C}$, top), indicating a lack of associative learning in the eyeblink conditioning task. GluA4-HET animals, on the other hand, were able to acquire associative memories similarly to WT (Figure S8). Analysis of CS-only trials revealed that, in contrast to WT animals, which had developed well-timed CRs by the end of training, GluA4-KO mice failed to show any learned eyelid closures in response to the CS (Figure 8D-E, top). To rule out the possibility that impaired vision was contributing to this effect (Gründer et al., 2000; Qin and Pourcho, 1999), we repeated the eyeblink conditioning experiments in a new set of mice using whisker stimulation as a CS. Again, conditioned responses were absent in GluA4-KO mice
(Figure 8B-E, bottom), demonstrating that GluA4 is required for eyeblink conditioning independent of CS modality. Thus, GluA4 appears to be dispensable for the control of coordinated locomotion (Figure 7), but is required for normal cerebellum-dependent associative learning.

\section{Discussion}

In the present study, we elucidated the functional significance of GluA4 in the cerebellum. Deletion of this AMPAR subunit strongly impaired both excitatory transmission at the cerebellum input layer and associative learning in adult mice. Despite compensatory enhancements in intrinsic excitability and NMDARmediated transmission that contributed to the residual function of GluA4-KO GCs, their spike output remained reduced. Computational modeling suggests that GluA4 facilitates pattern separation by the GC population and cerebellar learning, but does not influence population sparseness. On a behavioral level, even though locomotor coordination was largely intact in GluA4-KO mice, they were unable to form associative memories during eyeblink conditioning. Together, our results demonstrate that GluA4 is required for reliable synaptic excitation at the cerebellum input layer and for normal cerebellum-dependent associative learning.

\section{GluA4 Mediates Synaptic Excitation at the Cerebellar Input Layer}

Previous studies on GluA4 were mainly performed during development or in young animals (Fuchs et al., 2007; Huupponen et al., 2016; Yang et al., 2011; Zhu et al., 2000; but see: Seol and Kuner, 2015). GluA4 expression decreases during development (Zhao et al., 2019; Zhu et al., 2000). The cerebellar cortex, however, retains high 
levels of GluA4 in the adult (Keinänen et al., 1990; Monyer et al., 1991; Schwenk et al., 2014), primarily due to the numerous GCs expressing this AMPAR subunit. Here, we show that GluA4-containing AMPARs mediate the majority of excitatory input onto GCs and account for the fast kinetics of $\mathrm{MF} \rightarrow \mathrm{GC}$ EPSCs. These results provide additional evidence that the rapid kinetics of GluA4 are crucial for precise timing of postsynaptic spikes (Seol and Kuner, 2015; Yang et al., 2011). Overall, synapses with high GluA4 expression are capable of high-frequency transmission and exhibit strong short-term depression. GluA4 may thus be an integral part of the molecular framework enabling high-frequency information transfer in the mammalian brain.

\section{Compensations in GluA4-KO Mice}

The strongly diminished MF $\rightarrow$ GC EPSCs in GluA4-KO mice indicate that other AMPAR subunits cannot compensate for the loss of GluA4 (Yan et al., 2013; Yang et al., 2011). We did, however, observe a reduction of tonic inhibition in GluA4-KO GCs, which may enhance information flow through the cerebellar cortex (Hamann et al., 2002). Furthermore, increased NMDAR-EPSCs facilitated spiking of GluA4-KO GCs during high-frequency synaptic input. The larger NMDAR-EPSCs in GluA4-KO are most likely caused by increased glutamate release (Delvendahl et al., 2019), although we cannot exclude postsynaptic contributions. Together, attenuated inhibition and enhanced NMDAR-EPSCs contribute to synaptic information transfer by increasing the excitability of GluA4KO GCs. However, these adaptations are insufficient for maintaining the fidelity of GC output.

\section{A Low Number of Active GCs Can Sustain Normal Locomotion}

Despite the large reduction of $\mathrm{MF} \rightarrow \mathrm{GC}$ transmission, locomotor coordination was not impaired. This may point toward other pathways contributing to sensorimotor coordination or could be due to developmental compensation. It is intriguing to speculate that attenuated inhibition and enhanced non-AMPAR transmission sustain sufficient levels of $\mathrm{MF} \rightarrow \mathrm{GC}$ transmission in GluA4-KO animals to spare locomotor activity. The raised GC threshold might also be overcome by activation of multiple MF inputs, which can increase GC spike rates in vivo (Ishikawa et al., 2015). MF activity during locomotion is generally dense (Ishikawa et al., 2015; Knogler et al., 2017; Powell et al., 2015), which may activate a sufficient number of GCs for locomotor coordination in GluA4-KO mice. By contrast, the MF representation of the CS used for eyeblink conditioning is most likely not widespread and dense enough to activate a critical fraction of GCs in GluA4-KO mice (Giovannucci et al., 2017; Shimuta et al., 2020). Our finding that in the $\mathrm{KO}$ model, GC activity patterns promote faster learning than MF inputs only for high levels of MF activity might thus explain the discrepancy between spared locomotion and absence of associative learning in GluA4-
$\mathrm{KO}$ mice. These results are in line with previous studies, where impairments of $\mathrm{MF} \rightarrow \mathrm{GC}$ or $\mathrm{PF} \rightarrow \mathrm{PC}$ synapses did not cause locomotion defects but affected motor learning (Andreescu et al., 2011; Galliano et al., 2013; Peter et al., 2020; Seja et al., 2012). Indeed, a small number of active GCs may be sufficient for basic motor performance (Galliano et al., 2013; Schweighofer et al., 2001).

\section{The Role of MF $\rightarrow$ GC Synapses in Associative Learning}

Several previous studies investigated $P F \rightarrow P C$ synaptic function and plasticity in cerebellum-dependent motor learning (Galliano et al., 2013; Grasselli et al., 2020; Gutierrez-Castellanos et al., 2017; Peter et al., 2020; Wada et al., 2007). Afferent sensory information to the cerebellar cortex is first processed at the upstream $\mathrm{MF} \rightarrow \mathrm{GC}$ synapse (Billings et al., 2014; Cayco-Gajic et al., 2017), which may contribute to cerebellar learning. Indeed, disturbed AMPAR trafficking at $\mathrm{MF} \rightarrow \mathrm{GC}$ as well as other cerebellar synapses in ataxic stargazer mice compromises eyeblink conditioning (Hashimoto et al., 1999; Jackson and Nicoll, 2011). Our findings in GluA4-KO mice further support an essential role of $\mathrm{MF} \rightarrow \mathrm{GC}$ synapses in cerebellar associative learning. GluA4 is also expressed in Bergmann glia, but deletion of AMPARs in these cells does not affect eyeblink conditioning (Saab et al., 2012). Although we cannot rule out a contribution from other GluA4-containing synapses elsewhere in the brain, associative memory formation during eyeblink conditioning takes place within the cerebellum (Carey, 2011; De Zeeuw and Yeo, 2005; Freeman and Steinmetz, 2011; Heiney et al., 2014; Mauk, 1997; McCormick and Thompson, 1984), where GluA4 is predominantly expressed in GCs. Further, we show here that this AMPAR subunit is crucial for synaptic excitation of cerebellar GCs, but not of Purkinje and Golgi cells. Thus, we conclude that GluA4-containing AMPARs at MF $\rightarrow \mathrm{GC}$ synapses promote cerebellar associative learning. At this synaptic connection, the properties of the GluA4 AMPAR subunit are likely to be important for transmission and processing of the CS, which is conveyed by MF inputs (Albergaria et al., 2018; De Zeeuw and Yeo, 2005; Mauk, 1997).

\section{GluA4 Supports Computations Underlying Cerebellar Learning}

The remaining level of $\mathrm{GC}$ excitation in the absence of GluA4 seems sufficient for basic motor performance, but does not allow for associative learning. This indicates that associative memory formation may require more complex levels of GC activity and/or synaptic integration (Albergaria et al., 2018; Carey, 2011; Raymond and Medina, 2018). It is interesting to note that deletion of GluA4 not only caused a strong overall reduction in GC firing frequency, but also severely prolonged the first spike delay upon MF stimulation. GCs are subject to feedback- and feedforward inhibition by GoCs, which narrows the time window for GC integration to 5-10 ms (D'Angelo and De Zeeuw, 2009). The longer spike delay in GluA4-KO mice, 
together with the reduced $\mathrm{MF} \rightarrow \mathrm{GC}$ synaptic strength, raises the threshold of GC activation, leading to a loss of information (Billings et al., 2014; Cayco-Gajic et al., 2017). Our findings demonstrate that the higher GC threshold impaired expansion recoding of MF inputs onto the large population of GCs (Albus, 1971; Marr, 1969) (Figure 6G) by effectively reducing the coding space. By contrast, population sparseness of GCs was largely unaffected. Interestingly, the altered GC population coding was sufficient to dramatically impair pattern separation by the cerebellar input layer and learning in downstream circuits. These findings provide additional support for the hypothesis that pattern separation at the cerebellar input layer promotes associative memory formation.

The results we present here provide further evidence for the concept of an AMPAR code (Diering and Huganir, 2018), in which different AMPAR subunits serve different roles for synaptic function and learning. Future studies exploring the possible involvement of GluA4 in synaptic plasticity and other forms of learning will be an important component of our efforts to understand the rules governing cell-type specific expression and function of AMPAR subunits in the CNS.

\section{Materials and Methods Animals}

Animals were treated in accordance with national and institutional guidelines. All experiments were approved by the Cantonal Veterinary Office of Zurich (authorization no. ZH206/2016 and ZH009/2020) or by the Portuguese Direcção Geral de Veterinária (Ref. No. 0421/000/000/2015). GluA4-KO mice were kindly provided by $\mathrm{H}$. Monyer (Fuchs et al., 2007). GluA4-KO, GluA4-HET, and wild-type (WT) littermates were bred from heterozygous crosses. Genotyping of GluA4-KO mice was performed as described (Delvendahl et al., 2019). Experiments were performed in male and female mice, 1-5-month-old for slice recordings and 2-5-month-old for behavioral experiments. The animals were housed in groups of 3-5 in standard cages with food and water ad libitum; they were kept on a $12 \mathrm{~h}$-light/12h-dark cycle that was reversed for behavioral experiments.

\section{Electrophysiology}

Mice were sacrificed by rapid decapitation either without prior anesthesia or after isoflurane anesthesia in later experiments according to national guidelines. The cerebellar vermis was removed quickly and mounted in a chamber filled with cooled extracellular solution. $300-\mu \mathrm{m}$ thick parasagittal slices were cut using a Leica VT1200S vibratome (Leica Microsystems, Germany), transferred to an incubation chamber at $35^{\circ} \mathrm{C}$ for 30 minutes and then stored at room temperature until experiments. The extracellular solution (artificial cerebrospinal fluid, ACSF) for slice cutting and storage contained (in $\mathrm{mM}$ ): $125 \mathrm{NaCl}, 25 \mathrm{NaHCO}_{3}, 20$ D-glucose, $2.5 \mathrm{KCl}, 2 \mathrm{CaCl}_{2}, 1.25 \mathrm{NaH}_{2} \mathrm{PO}_{4}, 1 \mathrm{MgCl}_{2}$, bubbled with $95 \% \mathrm{O}_{2}$ and $5 \% \mathrm{CO}_{2}$. For recordings from Golgi cells, the slicing solution consisted of (in $\mathrm{mM}$ ): 230 sucrose, $25 \mathrm{D}$-glucose, 24 $\mathrm{NaHCO}_{3}, 4 \mathrm{MgCl}_{2}, 2.5 \mathrm{KCl}, 1.25 \mathrm{NaH}_{2} \mathrm{PO}_{4}, 0.5 \mathrm{CaCl}_{2}, 0.02$ D-APV.

Cerebellar slices were visualized using an upright microscope with a 60x, 1 NA water-immersion objective, infrared optics, and differential interference contrast (Scientifica, UK). The recording chamber was continuously perfused with ACSF supplemented with $10 \mu \mathrm{M}$ D-APV, $10 \mu \mathrm{M}$ bicuculline and $1 \mu \mathrm{M}$ strychnine unless otherwise stated. Voltage- and current-clamp recordings were done using a HEKA EPC10 amplifier (HEKA Elektronik $\mathrm{GmbH}$, Germany). Data were filtered at $10 \mathrm{kHz}$ and digitized with $100-200 \mathrm{kHz}$; recordings of spontaneous postsynaptic currents were filtered at $2.7 \mathrm{kHz}$ and digitized with $50 \mathrm{kHz}$. Experiments were performed at room temperature (21$25^{\circ} \mathrm{C}$ ). Patch pipettes were pulled to open-tip resistances of 3$8 \mathrm{M} \Omega$ (when filled with intracellular solution) from $1.5 \mathrm{~mm} / 0.86 \mathrm{~mm}$ (OD/ID) borosilicate glass (Science Products, Germany) using a DMZ puller (Zeitz Instruments, Germany).

The intracellular solution for EPSC and current-clamp recordings contained (in $\mathrm{mM}$ ): $150 \mathrm{~K}$-D-gluconate, $10 \mathrm{NaCl}, 10$ HEPES, 3 MgATP, 0.3 NaGTP, 0.05 ethyleneglycol-bis(2aminoethylether)-N,N,N',N'-tetraacetic acid (EGTA), pH adjusted to 7.3 using $\mathrm{KOH}$. Voltages were corrected for a liquid junction potential of $+13 \mathrm{mV}$.

We recorded in lobules III-VI of the cerebellar vermis. GC recordings were performed as described previously (Delvendahl et al., 2015, 2019). GC excitability was assessed in current-clamp mode by step current injections of 5-40 pA (duration, $200 \mathrm{~ms}$ ) from the resting membrane potential. Action potential firing was quantified over the full duration of current injection. GoCs were identified based upon their position in the GC layer, their large capacitance $(34.8 \pm 3.9 \mathrm{pF}, \mathrm{n}=29)$ and low-frequency of action potential firing (Dieudonne, 1995; Kanichay and Silver, 2008). Spontaneous EPSCs were recorded at a holding potential of $80 \mathrm{mV}$. MF $\rightarrow$ GoC EPSCs were recorded upon stimulation of the white matter or lower GC layer and identified based on the shortlatency and rapid kinetics of EPSCs and the absence of short-term facilitation (Kanichay and Silver, 2008) (Figure S1C). PF $\rightarrow$ GoC EPSCs were recorded upon stimulation of the molecular layer and displayed a longer, variable delay, slower kinetics, and pronounced short-term facilitation (Kanichay and Silver, 2008) (Figure S1D). Recordings from PCs were made similarly; $\mathrm{PF} \rightarrow \mathrm{PC}$ EPSCs were recorded upon stimulation of the molecular layer with increasing stimulation voltages $(3-18 \mathrm{~V})$ and spontaneous EPSCs were recorded at a holding potential of $-80 \mathrm{mV}$. NMDAR-mediated $\mathrm{MF} \rightarrow \mathrm{GC}$ EPSCs were recorded at a holding potential of $-80 \mathrm{mV}$ using a Mg-free extracellular solution supplemented with $10 \mu \mathrm{M}$ NBQX.

To record sIPSCs and tonic inhibitory currents in GCs, we used a CsCl-based intracellular solution containing (in $\mathrm{mM}$ ): 135 CsCl, 20 TEA-Cl, 10 HEPES, $5 \mathrm{Na}_{2}$ phosphocreatine, 4 MgATP, 0.3 NaGTP, 0.2 EGTA, pH adjusted to 7.3 using $\mathrm{CsOH}$ (liquid junction potential $\sim 0 \mathrm{mV}$ ). Due to the high intracellular [Cl-], IPSCs were recorded as inward currents at a holding potential of $-80 \mathrm{mV}$. $20 \mu \mathrm{M}$ GYKI-53655 were added to the extracellular solution to block AMPARs. To quantify tonic $\mathrm{GABA}_{\mathrm{A}} \mathrm{R}$-mediated conductance, recordings were made in the presence of $20 \mu \mathrm{M} \mathrm{GYKI-53655}$ and $1 \mu \mathrm{M}$ TTX. Following a stable baseline period, $20 \mu \mathrm{M}$ bicuculline were bath-applied. Tonic conductance was calculated from the difference in holding current before and after bicuculline application, and normalized to cell capacitance to account for account for cell-to-cell variability. GC whole-cell capacitance was not different between genotypes.

To study GC action potential firing upon high-frequency MF stimulation, membrane voltage was maintained at $-70 \mathrm{mV}$ (Rothman et al., 2009) by current injection. Similar results were obtained at a membrane potential of $-80 \mathrm{mV}$ (Figure S3A). Ten MF stimuli were applied at frequencies of $100-300 \mathrm{~Hz}$. GC spikes were detected using a threshold of $-20 \mathrm{mV}$ and the spike frequency was calculated over the entire train duration. 


\section{Western Blotting}

Cerebellar tissue was rapidly dissected and frozen at $-80^{\circ} \mathrm{C}$. Samples were lysed and sonicated in RIPA buffer with protein inhibitors (Roche). Protein concentrations were estimated by BCA assay (Thermo Fisher Scientific) according to manufacturer's instructions. Protein extracts were then denatured with $2 \times$ Laemmi buffer and boiled at $95^{\circ} \mathrm{C}$ for $5 \mathrm{~min}$. Samples with equal amount of protein were run on $10 \%$ SDS-polyarylamide gel, and then transferred onto PVDF membrane (Thermo Fisher Scientific). Membranes were cut horizontally at appropriate molecular weights and blocked in $5 \%$ milk $(\mathrm{w} / \mathrm{v})$ in $1 \times$ PBS for $1 \mathrm{~h}$ on a shaker. Blots were then incubated with primary antibodies: anti-GluA4 (1:100, Santa Cruz Biotechnology) and anti- $\beta$-Tubulin $(1: 1,000$, DSHB) overnight at $4{ }^{\circ} \mathrm{C}$. To detect transferred proteins, we used horseradish peroxide-conjugated goat anti-mouse antibody (1:1,000, Jackson Immuno Research). Blots were developed with a chemiluminescence detection kit (Thermo Fisher Scientific) and images were acquired with Fusion SL (Vilber). Band intensities were quantified using ImageJ software and normalized to $\beta$ Tubulin signal.

\section{MF $\rightarrow$ GC Modeling}

A simple GC adaptive exponential integrate-and-fire model (Brette and Gerstner, 2005) was implemented and run using Neuromatic (Rothman and Silver, 2018) in Igor Pro (Wavemetrics) with a fixed time step of $50 \mu \mathrm{s}$. Model parameters were constrained using our experimental data or taken from the literature. To model $\mathrm{MF} \rightarrow \mathrm{GC}$ synapses, population averages of 100-Hz AMPAR- and NMDAR-trains were converted into synaptic conductance with direct and spillover components (Rothman et al., 2009). We optimized the model $G_{A M P A}$ and $G_{\text {NMDA }}$ parameters to fit the synaptic conductance during $100-\mathrm{Hz}$ trains. Short-term plasticity was implemented using an $\mathrm{R}^{\star} \mathrm{P}$ model (Rothman and Silver, 2018) and parameters were fine-tuned using $100-\mathrm{Hz}$ and $300-\mathrm{Hz}$ population average data for each genotype. Four independent Poisson spike trains were applied to the GC model, with a sum of $10-1000 \mathrm{~Hz}$. Qualitatively similar results were obtained with lower fractions of active MFs. Duration of the spike trains was $1 \mathrm{~s}$ and each MF input had a refractory time of $0.6 \mathrm{~ms}$ (Ritzau-Jost et al., 2014). Simulations were performed for $37^{\circ} \mathrm{C}$ and resting membrane potential was set at $-80 \mathrm{mV}$. The average model spiking frequency was calculated from simulating ten independent runs for each frequency. Plots of GC firing versus MF stimulation frequency were fit with a Hill equation:

$$
F(x)=\frac{F_{\max }}{1+\left(\frac{x_{1 / 2}}{x}\right)^{n}}
$$

where $F_{\max }$ is the maximum firing rate, $x_{1 / 2}$ the MF stimulation frequency at which $F$ reaches half maximum and $n$ the exponent factor. The offset was calculated taken from $x_{1 / 2}$ of the fits; the gain was calculated from the slope of the fits between $2 \%$ and $60 \%$ of the maximum (Rothman et al., 2009).

\section{Feedforward GC Layer Network Modeling}

We adapted the model described in (Cayco-Gajic et al., 2017) to study the effect of GluA4-KO on the function of the cerebellar input layer. In the model, $187 \mathrm{MFs}$ are connected to 487 GCs in a sphere of $80 \mu \mathrm{m}$ diameter; neuron numbers and connectivity are based on anatomical data. The feedforward model was presented with $n=640$ different MF input patterns ( $50 \mathrm{~Hz}$ firing, sampled from a Poisson distribution) and GC activity (i.e. spike counts in a $30-\mathrm{ms}$ time window) was extracted. A perceptron algorithm was subsequently used to classify MF and GC activity patterns into 10 random classes (Cayco-Gajic et al., 2017). We compared the published model parameters (Cayco-
Gajic et al., 2017) against a model with scaled $\mathrm{G}_{\text {AMPA }}$ and $\mathrm{G}_{\text {NMDA }}$ corresponding to the observed relative changes in GluA4-KO GCs (scaling factors 0.2 and 1.2 , respectively, Figures $1 \mathrm{C}$ and $5 \mathrm{~B}$ ). In addition, the input conductance of the GluA4-KO model was reduced by $0.16 \mathrm{nS}$ (according to Figure $2 \mathrm{G}$ ). Learning speed, population sparseness and total variance were calculated and analyzed as described in (Cayco-Gajic et al., 2017). In brief, learning speed was analyzed as inverse of the number of training epochs needed to reach a root-mean-square error of 0.2 . Population sparseness was calculated as:

$$
\frac{N-\frac{\left(\sum_{i} x_{i}\right)^{2}}{\sum_{i} x_{i}^{2}}}{N-1}
$$

where $N$ is the number of GCs and $x_{i}$ is the ith GC's spike count. Sparseness was averaged over all $n$ activity patterns. Total variance was calculated as the sum of all variances:

$$
\sum_{i} \operatorname{var}\left(\boldsymbol{v}_{\boldsymbol{i}}\right)
$$

where $\boldsymbol{v}_{\boldsymbol{i}}$ is a vector of length $n$ consisting of the ith GC's spike counts for all $n$ activity patterns.

\section{Delay Eyeblink Conditioning}

Animals were anesthetized with isoflurane (4\% induction and $0.5-1 \%$ for maintenance), placed in a stereotaxic frame (David Kopf Instruments, Tujunga, CA) and a head plate was glued to the skull with dental cement (Super Bond - C\&B). After the surgery, mice were monitored and allowed at least 2 days of recovery.

All eyeblink conditioning experiments were run on a motorized treadmill, as described in previous work (Albergaria et al., 2018). Briefly, head-fixed mice were habituated to the motorized behavioral setup for at least 2 days prior to training. The speed of the treadmill was set to $0.12 \mathrm{~m} / \mathrm{s}$ using a DC motor with an encoder (Maxon). After habituation, each training session consisted of 100 trials, separated by a randomized inter trial interval of 10-15 s. In each trial, CS and US onsets were separated by a fixed interval of $300 \mathrm{~ms}$ and both stimuli co-terminated.

For all training experiments, the unconditioned stimulus (US) was an air-puff (30-50 psi, $50 \mathrm{~ms})$ controlled by a Picospritzer (Parker) and was adjusted for each session of each mouse so that the unconditioned stimulus elicited a full eye blink. The CS had a $350 \mathrm{~ms}$ duration and was either i) a white light LED positioned $\sim 3 \mathrm{~cm}$ directly in front of the mouse or ii) a piezoelectric device placed $\sim 0.5 \mathrm{~cm}$ away from the left vibrissal pad.

Eyelid movements of the right eye were recorded using a high-speed monochromatic camera (Genie HM640, Dalsa) to monitor a $172 \times 160$ pixel region, at $900 \mathrm{fps}$. Custom-written software using LabVIEW, together with a NI PCIE-8235 frame grabber and a NI-DAQmx board (National Instruments), was used to trigger and control all the hardware in a synchronized manner.

\section{Locomotion Analysis}

We used the LocoMouse system to quantify overground locomotion (Machado et al., 2015). Animals run back and forward between two dark boxes, in a glass corridor $(66.5 \mathrm{~cm}$ long and $4.5 \mathrm{~cm}$ wide with a mirror placed at $45^{\circ}$ under). Individual trials consisted of single crossings of the corridor. A single high-speed camera (AVT Bonito, $1440 \times 250$ pixels, $400 \mathrm{fps}$ ) recorded both bottom and side views of walking mice during the trial. Animals were acclimated to the overground setup for several sessions before data collection; no food or water restriction or reward was used.

Homozygous GluA4-KOs ranged in size from $16-37 \mathrm{~g}$. Sizematched littermate HET (18-38 g) and WT animals (16-38 g) were selected as controls (Machado et al., 2015). Ten to twenty- 
five corridor trials were collected in each session for five consecutive days. An average of $1207 \pm 203$ strides were collected per homozygous GluA4 mouse $(310 \pm 51$ strides per animal per paw), $856 \pm 342$ strides per heterozygous GluA4 mouse $(219 \pm 94$ strides per animal per paw) and $840 \pm 278$ strides per WT mouse (218 \pm 70 strides per animal per paw) were collected.

\section{Data Analysis}

Electrophysiological data were analyzed using customwritten routines or Neuromatic (Rothman and Silver, 2018) in Igor Pro (Wavemetrics, USA). EPSC amplitudes were quantified as difference between peak and baseline. Decay kinetics were analyzed by fitting a bi-exponential function to the EPSC decay time course. To detect spontaneous or miniature postsynaptic currents, we used a template-matching routine (Rothman and Silver, 2018). Detected events were analyzed as described above. Only cells with $>10$ detected spontaneous events were included for amplitude quantification.

Simulation results were analyzed using Igor Pro and Python. To calculate the van Rossum distance, pre- and postsynaptic spike trains were convolved using a 10-ms exponential kernel. The area of the squared difference was then multiplied by the inverse of the kernel time constant to give the van Rossum distance. Spike synchronization was calculated using PySpike (Mulansky and Kreuz, 2016).

For eyeblink conditioning, video from each trial were analyzed offline with custom-written software using MATLAB (MathWorks). The distance between eyelids was calculated frame by frame by thresholding the grayscale image of the eye and extracting the count of pixels that constitute the minor axis of the elliptical shape that delineates the eye. Eyelid traces were normalized for each session, ranging from 1 (full blink) to 0 (eye fully open). Trials were classified as CRs if the eyelid closure reached at least 0.1 (in normalized pixel values) and occurred between $100 \mathrm{~ms}$ after the time of CS onset and the onset of US.

Analysis of locomotion data was performed in Matlab $2012 \mathrm{~b}$ and 2015a. Paw, nose and tail tracks $(\mathrm{x}, \mathrm{y}, \mathrm{z})$ were obtained from the LocoMouse tracker (Machado et al., 2015) (https://github.com/careylab/LocoMouse). All tracks were divided in stride cycles and the data was sorted into speed bins $(0.05 \mathrm{~m} / \mathrm{s}$ bin width). Gait parameters (individual limb movements and interlimb coordination) were calculated as follows:

\section{Individual limb}

Walking speed: $x$ displacement of the body center during that stride divided by the stride duration

Stride length: $x$ displacement from touchdown to touchdown of single limb

Stride duration: time between two consecutive stance onsets

Cadence: inverse of stride duration

Swing velocity: $x$ displacement of single limb during swing phase divided by swing duration

Stance duration: time in milliseconds that foot is on the ground during stride

Duty factor: stance duration divided by stride duration

Trajectories: $(x, y, z)$ trajectories were aligned to swing onset and resampled to 100 equidistant points using linear interpolation. Interpolated trajectories were then binned by speed and the average trajectory was computed for each individual animal and smoothed with a Savitzky-Golay first-order filter with a 3-point window size.

Instantaneous swing velocity: the derivative of swing trajectory
Interlimb and whole-body coordination

Stance phase: relative timing of limb touchdowns to stride cycle of reference paw (FR). Calculated as: (stance time - stance time reference paw) / stride duration.

Base of support: width between the two front and two hind paws during stance phase

Body y displacement: y displacement of the body center during that stride

Supports: Support types were categorized by how many and which paws were on the ground, expressed as a percentage of the total stride duration for each stride. Paw support categories include 3paw, 2-paw diagonal, 2-paw other/non-diagonal (homolateral and homologous), and 2-paw front (only) supports.

Double support for each limb is defined as the percentage of the stride cycle between the touch down of a reference paw to lift-off of the contralateral paw. Because at higher speeds (running), the opposing limb lifts off before the reference paw touches down, we included negative double support by looking backwards in time, up to $25 \%$ of the stride cycle duration. Positive values of double support indicate that contralateral lift-off occurred after reference paw touch down, and negative values indicate that contralateral lift-off occurred before reference paw touch down. Note that front paw double support percentages include 2-paw front (only) support patterns as well as 3- and 4-paw support patterns in which both front paws were on the ground.

Tail and nose phases: For each speed bin we correlate the stridewise tail and nose trajectories with the trajectory given by the difference between the forward position of the right paw and the forward position of the left paw (also normalized to the stride). The phase is then calculated by the delay in which this correlation is maximized.

Tail and nose peak-to-peak amplitude: the change between peak (highest amplitude value) and trough (lowest amplitude value) in y or $z$ during a stride duration.

Variability: All variability analyses were based on coefficients of variation (CV).

\section{Principal Component and Linear Discriminant Analyses}

The locomotor dataset consisted of a matrix of 45 features for each mouse and speed bin. Many gait features are highly correlated with speed (Machado et al., 2015), so to avoid intervariable correlation and overfitting we first performed PCA. The first 10 principal components explained more than $85 \%$ of the variance and the data projected onto these 10 principal components were used as input to the LDA (Machado et al., 2020). LDA output is displayed separately for each speed bin to verify that the pattern of differences across groups was captured across all walking speeds.

\section{Statistical Analysis}

Electrophysiological data were analyzed using ANOVA and two-tailed t-tests. Statistical testing was performed in R. Data in figures are presented as mean \pm standard error of the mean (SEM). Eyeblink conditioning data were analyzed using t-tests. Locomotion data were analyzed in Matlab with the Statistics toolbox. An independent samples t-test was used to test for differences in walking speed distributions (Figure S6A). For all other gait parameters, analysis was performed on animal averages binned by speed using mixed effects models (Bates et al., 2015). Fixed-effects terms included speed and genotype; animals were included as random terms. Table S1 reports effects of genotype for all locomotor measurements as $F$ statistics from mixed ANOVAs with Satterthwaite degrees of freedom correction. No corrections were made for multiple comparisons. 
bioRxiv preprint doi: https://doi.org/10.1101/2020.12.04.412023; this version posted December 4, 2020. The copyright holder for this preprint (which was not certified by peer review) is the author/funder, who has granted bioRxiv a license to display the preprint in perpetuity. It is made available under aCC-BY-NC-ND 4.0 International license.

\section{References}

Aizenman, C.D., and Linden, D.J. (2000). Rapid, synaptically driven increases in the intrinsic excitability of cerebellar deep nuclear neurons. Nat. Neurosci. 3 , 109-111.

Albergaria, C., Silva, N.T., Pritchett, D.L., and Carey, M.R. (2018). Locomotor activity modulates associative learning in mouse cerebellum. Nat. Neurosci. 21, 725-735.

Albus, J.S. (1971). A theory of cerebellar function. Math. Biosci. 10, 25-61. Andreescu, C.E., Prestori, F., Brandalise, F., D'Errico, A., De Jeu, M.T.G., Rossi, P., Botta, L., Kohr, G., Perin, P., D'Angelo, E., et al. (2011). NR2A subunit of the Nmethyl $d$-aspartate receptors are required for potentiation at the mossy fiber to granule cell synapse and vestibulo-cerebellar motor learning. Neuroscience 176 274-283.

Arenz, A., Silver, R.A., Schaefer, A.T., and Margrie, T.W. (2008). The Contribution of Single Synapses to Sensory Representation in Vivo. Science 321, 977-980. Bates, D., Mächler, M., Bolker, B., and Walker, S. (2015). Fitting Linear MixedEffects Models Using Ime4. J. Stat. Softw. 67.

Billings, G., Piasini, E., Lorincz, A., Nusser, Z., and Silver, R.A. (2014). Network Structure within the Cerebellar Input Layer Enables Lossless Sparse Encoding. Neuron 83, 960-974.

Brette, R., and Gerstner, W. (2005). Adaptive Exponential Integrate-and-Fire Model as an Effective Description of Neuronal Activity. J. Neurophysiol. 94 3637-3642.

Brickley, S.G., Revilla, V., Cull-Candy, S.G., Wisden, W., and Farrant, M. (2001). Adaptive regulation of neuronal excitability by a voltage-independent potassium conductance. Nature 409, 88-92.

Carey, M.R. (2011). Synaptic mechanisms of sensorimotor learning in the cerebellum. Curr. Opin. Neurobiol. 21, 609-615.

Cathala, L., Holderith, N.B., Nusser, Z., DiGregorio, D.A., and Cull-Candy, S.G. (2005). Changes in synaptic structure underlie the developmental speeding of AMPA receptor-mediated EPSCs. Nat. Neurosci. 8, 1310-1318.

Cayco-Gajic, N.A., and Silver, R.A. (2019). Re-evaluating Circuit Mechanisms Underlying Pattern Separation. Neuron 101, 584-602.

Cayco-Gajic, N.A., Clopath, C., and Silver, R.A. (2017). Sparse synaptic connectivity is required for decorrelation and pattern separation in feedforward networks. Nat. Commun. 8, 1116.

Chadderton, P., Margrie, T.W., and Häusser, M. (2004). Integration of quanta in cerebellar granule cells during sensory processing. Nature 428, 856-860.

Citri, A., Bhattacharyya, S., Ma, C., Morishita, W., Fang, S., Rizo, J., and Malenka, R.C. (2010). Calcium Binding to PICK1 Is Essential for the Intracellular Retention of AMPA Receptors Underlying Long-Term Depression. J. Neurosci. 30, 1643716452.

D'Angelo, E., and De Zeeuw, C.I. (2009). Timing and plasticity in the cerebellum: focus on the granular layer. Trends Neurosci. 32, 30-40.

D’Angelo, E., De Filippi, G., Rossi, P., and Taglietti, V. (1995). Synaptic excitation of individual rat cerebellar granule cells in situ: evidence for the role of NMDA receptors. J. Physiol. 484, 397-413.

De Zeeuw, C.I., and Yeo, C.H. (2005). Time and tide in cerebellar memory formation. Curr. Opin. Neurobiol. 15, 667-674.

Delvendahl, I., and Hallermann, S. (2016). The Cerebellar Mossy Fiber Synapse as a Model for High-Frequency Transmission in the Mammalian CNS. Trends Neurosci. 39, 722-737.

Delvendahl, I., Straub, I., and Hallermann, S. (2015). Dendritic patch-clamp recordings from cerebellar granule cells demonstrate electrotonic compactness. Front. Cell. Neurosci. 9, 796-798.

Delvendahl, I., Kita, K., and Müller, M. (2019). Rapid and sustained homeostatic control of presynaptic exocytosis at a central synapse. Proc. Natl. Acad. Sci. 116, 23783-23789.

Desai, N.S., Rutherford, L.C., and Turrigiano, G.G. (1999). Plasticity in the intrinsic excitability of cortical pyramidal neurons. Nat. Neurosci. 2, 515-520.

Diedrichsen, J., King, M., Hernandez-Castillo, C., Sereno, M., and Ivry, R.B. (2019) Universal Transform or Multiple Functionality? Understanding the Contribution of the Human Cerebellum across Task Domains. Neuron 102, 918-928.

Diering, G.H., and Huganir, R.L. (2018). The AMPA Receptor Code of Synaptic Plasticity. Neuron 100, 314-329.

Dieudonne, S. (1995). Glycinergic synaptic currents in Golgi cells of the rat cerebellum. Proc. Natl. Acad. Sci. 92, 1441-1445.

DiGregorio, D.A., Nusser, Z., and Silver, R.A. (2002). Spillover of glutamate onto synaptic AMPA receptors enhances fast transmission at a cerebellar synapse. Neuron 35, 521-533.

Duguid, I., Branco, T., Chadderton, P., Arlt, C., Powell, K., and Häusser, M. (2015) Control of cerebellar granule cell output by sensory-evoked Golgi cell inhibition. Proc. Natl. Acad. Sci. 112, 13099-13104.

Freeman, J.H., and Steinmetz, A.B. (2011). Neural circuitry and plasticity mechanisms underlying delay eyeblink conditioning. Learn. Mem. 18, 666-677. Fuchs, E.C., Zivkovic, A.R., Cunningham, M.O., Middleton, S., LeBeau, F.E.N., Bannerman, D.M., Rozov, A., Whittington, M.A., Traub, R.D., Rawlins, J.N.P., et al (2007). Recruitment of Parvalbumin-Positive Interneurons Determines Hippocampal Function and Associated Behavior. Neuron 53, 591-604.
Galliano, E., Gao, Z., Schonewille, M., Todorov, B., Simons, E., Pop, A.S., D'Angelo, E., van den Maagdenberg, A.M.J.M., Hoebeek, F.E., and De Zeeuw, C.I. (2013). Silencing the Majority of Cerebellar Granule Cells Uncovers Their Essential Role in Motor Learning and Consolidation. Cell Rep. 3, 1239-1251.

Geiger, J., Melcher, T., Koh, D.S., and Sakmann, B. (1995). Relative abundance of subunit mRNAs determines gating and $\mathrm{Ca}^{2+}$ permeability of AMPA receptors in principal neurons and interneurons in rat CNS. Neuron 15, 193-204.

Giovannucci, A., Badura, A., Deverett, B., Najafi, F., Pereira, T.D., Gao, Z., Ozden, I., Kloth, A.D., Pnevmatikakis, E., Paninski, L., et al. (2017). Cerebellar granule cells acquire a widespread predictive feedback signal during motor learning. Nat. Neurosci. 20, 727-734.

Grasselli, G., Boele, H.-J., Titley, H.K., Bradford, N., van Beers, L., Jay, L., Beekhof, G.C., Busch, S.E., De Zeeuw, C.I., Schonewille, M., et al. (2020). SK2 channels in cerebellar Purkinje cells contribute to excitability modulation in motor-learningspecific memory traces. PLOS Biol. 18, e3000596.

Gründer, T., Kohler, K., and Guenther, E. (2000). Distribution and Developmental Regulation of AMPA Receptor Subunit Proteins in Rat Retina. Invest. Ophthalmol. Vis. Sci. 41, 7.

Gutierrez-Castellanos, N., Da Silva-Matos, C.M., Zhou, K., Canto, C.B., Renner, M.C., Koene, L.M.C., Ozyildirim, O., Sprengel, R., Kessels, H.W., and De Zeeuw, C.I. (2017). Motor Learning Requires Purkinje Cell Synaptic Potentiation through Activation of AMPA-Receptor Subunit GluA3. Neuron 93, 409-424.

Hamann, M., Rossi, D.J., and Attwell, D. (2002). Tonic and Spillover Inhibition of Granule Cells Control Information Flow through Cerebellar Cortex. Neuron 33, 625-633.

Hashimoto, K., Fukaya, M., Qiao, X., Sakimura, K., Watanabe, M., and Kano, M. (1999). Impairment of AMPA receptor function in cerebellar granule cells of ataxic mutant mouse stargazer. J. Neurosci. 19, 6027-6036.

Heck, D.H., De Zeeuw, C.I., Jaeger, D., Khodakhah, K., and Person, A.L. (2013). The Neuronal Code(s) of the Cerebellum. J. Neurosci. 33, 17603-17609. Heiney, S.A., Wohl, M.P., Chettih, S.N., Ruffolo, L.I., and Medina, J.F. (2014). Cerebellar-Dependent Expression of Motor Learning during Eyeblink Conditioning in Head-Fixed Mice. J. Neurosci. 34, 14845-14853.

Hollmann, M., and Heinemann, S. (1994). Cloned Glutamate Receptors. Annu. Rev. Neurosci. 17, 31-108.

Huupponen, J., Atanasova, T., Taira, T., and Lauri, S.E. (2016). GluA4 subunit of AMPA receptors mediates the early synaptic response to altered network activity in the developing hippocampus. J. Neurophysiol. 115, 2989-2996. Ishikawa, T., Shimuta, M., and Häusser, M. (2015). Multimodal sensory integration in single cerebellar granule cells in vivo. ELife 4, 25-26.

Jackson, A.C., and Nicoll, R.A. (2011). Stargazin (TARP Y-2) Is Required for Compartment-Specific AMPA Receptor Trafficking and Synaptic Plasticity in Cerebellar Stellate Cells. J. Neurosci. 31, 3939-3952.

van Kan, P.L., Gibson, A.R., and Houk, J.C. (1993). Movement-related inputs to intermediate cerebellum of the monkey. J. Neurophysiol. 69, 74-94.

Kanichay, R.T., and Silver, R.A. (2008). Synaptic and Cellular Properties of the Feedforward Inhibitory Circuit within the Input Layer of the Cerebellar Cortex. J. Neurosci. 28, 8955-8967.

Karmarkar, U.R., and Buonomano, D.V. (2006). Different forms of homeostatic plasticity are engaged with distinct temporal profiles: Differential expression of homeostatic plasticity. Eur. J. Neurosci. 23, 1575-1584.

Keinänen, K., Wisden, W., Sommer, B., Werner, P., Herb, A., Verdoorn, T., Sakmann, B., and Seeburg, P. (1990). A family of AMPA-selective glutamate receptors. Science $249,556-560$.

Knogler, L.D., Markov, D.A., Dragomir, E.I., Štih, V., and Portugues, R. (2017). Sensorimotor Representations in Cerebellar Granule Cells in Larval Zebrafish Are Dense, Spatially Organized, and Non-temporally Patterned. Curr. Biol. 27, 12881302.

Machado, A.S., Darmohray, D.M., Fayad, J., Marques, H.G., and Carey, M.R. (2015). A quantitative framework for whole-body coordination reveals specific deficits in freely walking ataxic mice. ELife 4, e07892.

Machado, A.S., Marques, H.G., Duarte, D.F., Darmohray, D.M., and Carey, M.R. (2020). Shared and specific signatures of locomotor ataxia in mutant mice. ELife 9, e55356.

Marr, D. (1969). A theory of cerebellar cortex. J. Physiol. 202, 437-470.

Mauk, M.D. (1997). Roles of Cerebellar Cortex and Nuclei in Motor Learning: Contradictions or Clues? Neuron 18,343-346.

McCormick, D., and Thompson, R. (1984). Cerebellum: essential involvement in the classically conditioned eyelid response. Science 223, 296-299.

Mitchell, S.J., and Silver, R.A. (2003). Shunting inhibition modulates neuronal gain during synaptic excitation. Neuron $38,433-445$.

Monyer, H., Seeburg, P.H., and Wisden, W. (1991). Glutamate-operated channels: Developmentally early and mature forms arise by alternative splicing. Neuron 6 , 799-810.

Morton, S., and Bastian, A. (2007). Mechanisms of cerebellar gait ataxia. The Cerebellum 6, 79-86

Mosbacher, J., Schöpfer, R., and Monyer, H. (1994). A molecular determinant for submillisecond desensitization in glutamate receptors. Science $266,1059-$ 1062. 
bioRxiv preprint doi: https://doi.org/10.1101/2020.12.04.412023; this version posted December 4, 2020. The copyright holder for this preprint (which was not certified by peer review) is the author/funder, who has granted bioRxiv a license to display the preprint in perpetuity. It is made available under aCC-BY-NC-ND 4.0 International license.

Mulansky, M., and Kreuz, T. (2016). PySpike-A Python library for analyzing spike train synchrony. SoftwareX 5, 183-189.

Mulansky, M., Bozanic, N., Sburlea, A., and Kreuz, T. (2015). A guide to timeresolved and parameter-free measures of spike train synchrony. 2015 Int. Conf. Event-Based Control Commun. Signal Process. EBCCSP 1-8.

Osborne, L.C., Hohl, S.S., Bialek, W., and Lisberger, S.G. (2007). Time Course of Precision in Smooth-Pursuit Eye Movements of Monkeys. J. Neurosci. 27, 2987-

Paz, J.T., Bryant, A.S., Peng, K., Fenno, L., Yizhar, O., Frankel, W.N., Deisseroth, K. and Huguenard, J.R. (2011). A new mode of corticothalamic transmission revealed in the $\mathrm{Gria}^{-/-}$model of absence epilepsy. Nat. Neurosci. 14, 11671173.

Peter, S., Urbanus, B.H.A., Klaassen, R.V., Wu, B., Boele, H.-J., Azizi, S., Slotman, J.A., Houtsmuller, A.B., Schonewille, M., Hoebeek, F.E., et al. (2020). AMPAR Auxiliary Protein SHISA6 Facilitates Purkinje Cell Synaptic Excitability and Procedural Memory Formation. Cell Rep. 31, 107515.

Powell, K., Mathy, A., Duguid, I., and Häusser, M. (2015). Synaptic representation of locomotion in single cerebellar granule cells. ELife 4, e07290.

Qin, P., and Pourcho, R.G. (1999). AMPA-selective glutamate receptor subunits GluR2 and GluR4 in the cat retina: An immunocytochemical study. Vis. Neurosci. $16,1105-1114$

Rancz, E.A., Ishikawa, T., Duguid, I., Chadderton, P., Mahon, S., and Häusser, M. (2007). High-fidelity transmission of sensory information by single cerebellar mossy fibre boutons. Nature 450, 1245-1248.

Raymond, J.L., and Medina, J.F. (2018). Computational Principles of Supervised Learning in the Cerebellum. Annu. Rev. Neurosci. 41, 233-253.

Renner, M.C., Albers, E.H., Gutierrez-Castellanos, N., Reinders, N.R., van Huijstee, A.N., Xiong, H., Lodder, T.R., and Kessels, H.W. (2017). Synaptic plasticity through activation of GluA3-containing AMPA-receptors. ELife 6, e25462.

Ritzau-Jost, A., Delvendahl, I., Rings, A., Byczkowicz, N., Harada, H., Shigemoto, R., Hirrlinger, J., Eilers, J., and Hallermann, S. (2014). Ultrafast Action Potentials Mediate Kilohertz Signaling at a Central Synapse. Neuron 84, 152-163.

Roth, R.H., Cudmore, R.H., Tan, H.L., Hong, I., Zhang, Y., and Huganir, R.L. (2020) Cortical Synaptic AMPA Receptor Plasticity during Motor Learning. Neuron 105 895-908.

Rothman, J.S., and Silver, R.A. (2014). Data-Driven Modeling of Synaptic Transmission and Integration. In Progress in Molecular Biology and Translational Science, (Elsevier), pp. 305-350.

Rothman, J.S., and Silver, R.A. (2018). NeuroMatic: An Integrated Open-Source Software Toolkit for Acquisition, Analysis and Simulation of Electrophysiological Data. Front. Neuroinformatics 12, 1159-21.

Rothman, J.S., Cathala, L., Steuber, V., and Silver, R.A. (2009). Synaptic depression enables neuronal gain control. Nature 457, 1015-1018.

Rudolph, S., Guo, C., Pashkovski, S.L., Osorno, T., Gillis, W.F., Krauss, J.M., Nyitrai, H., Flaquer, I., El-Rifai, M., Datta, S.R., et al. (2020). Cerebellum-Specific Deletion of the GABAA Receptor $\delta$ Subunit Leads to Sex-Specific Disruption of Behavior. Cell Rep. 33, 108338.

Saab, A.S., Neumeyer, A., Jahn, H.M., Cupido, A., Šimek, A.A.M., Boele, H.-J., Scheller, A., Le Meur, K., Götz, M., Monyer, H., et al. (2012). Bergmann glial AMPA receptors are required for fine motor coordination. Science 337, 749-753.

Schweighofer, N., Doya, K., and Lay, F. (2001). Unsupervised learning of granule cell sparse codes enhances cerebellar adaptive control. Neuroscience 103,3550.

Schwenk, J., Baehrens, D., Haupt, A., Bildl, W., Boudkkazi, S., Roeper, J., Fakler, B. and Schulte, U. (2014). Regional Diversity and Developmental Dynamics of the AMPA-Receptor Proteome in the Mammalian Brain. Neuron 84, 41-54.
Seja, P., Schonewille, M., Spitzmaul, G., Badura, A., Klein, I., Rudhard, Y., Wisden, W., Hübner, C.A., De Zeeuw, C.I., and Jentsch, T.J. (2012). Raising cytosolic $\mathrm{Cl}^{-}$in cerebellar granule cells affects their excitability and vestibulo-ocular learning: Intraneuronal chloride and cerebellar function. EMBO J. 31, 1217-1230.

Seol, M., and Kuner, T. (2015). Ionotropic glutamate receptor GluA4 and T-type calcium channel Cav3.1 subunits control key aspects of synaptic transmission at the mouse L5B-POm giant synapse. Eur. J. Neurosci. 42, 3033-3044.

Shi, S.-H., Hayashi, Y., Esteban, J.A., and Malinow, R. (2001). Subunit-Specific Rules Governing AMPA Receptor Trafficking to Synapses in Hippocampal Pyramidal Neurons. Cell 105, 331-343.

Shimuta, M., Sugihara, I., and Ishikawa, T. (2020). Multiple signals evoked by unisensory stimulation converge onto cerebellar granule and Purkinje cells in mice. Commun. Biol. 3, 381

Silva, M.M., Rodrigues, B., Fernandes, J., Santos, S.D., Carreto, L., Santos, M.A.S., Pinheiro, P., and Carvalho, A.L. (2019). MicroRNA-186-5p controls GluA2 surface expression and synaptic scaling in hippocampal neurons. Proc. Natl. Acad. Sci. $116,5727-5736$

Sjöstedt, E., Zhong, W., Fagerberg, L., Karlsson, M., Mitsios, N., Adori, C., Oksvold P.. Edfors, F., Limiszewska, A., Hikmet, F., et al. (2020). An atlas of the proteincoding genes in the human, pig, and mouse brain. Science 367 , eaay5947.

Steinberg, J.P., Takamiya, K., Shen, Y., Xia, J., Rubio, M.E., Yu, S., Jin, W., Thomas, G.M., Linden, D.J., and Huganir, R.L. (2006). Targeted In Vivo Mutations of the AMPA Receptor Subunit GluR2 and Its Interacting Protein PICK1 Eliminate Cerebellar Long-Term Depression. Neuron 49, 845-860.

Stell, B.M., Brickley, S.G., Tang, C.Y., Farrant, M., and Mody, I. (2003). Neuroactive steroids reduce neuronal excitability by selectively enhancing tonic inhibition mediated by delta subunit-containing GABAA receptors. Proc. Natl. Acad. Sci. U. S. A. $100,14439-14444$.

Swanson, G.T. Kamboj, S.K and Cull-Candy, S.G. (1997). Single-channel properties of recombinant AMPA receptors depend on RNA editing, splice variation, and subunit composition. J. Neurosci. 17, 58-69.

Traynelis, S.F., Wollmuth, L.P., McBain, C.J., Menniti, F.S., Vance, K.M., Ogden, K.K., Hansen, K.B., Yuan, H., Myers, S.J., and Dingledine, R. (2010). Glutamate Receptor Ion Channels: Structure, Regulation, and Function. Pharmacol. Rev. 62 405-496.

Wada, N., Kishimoto, Y, Watanabe, D., Kano, M., Hirano, T., Funabiki, K., and Nakanishi, S. (2007). Conditioned eyeblink learning is formed and stored without cerebellar granule cell transmission. Proc. Natl. Acad. Sci. 104, 16690-16695. Witter, L., and De Zeeuw, C.I. (2015). Regional functionality of the cerebellum. Curr. Opin. Neurobiol. 33, 150-155

Yan, D., Yamasaki, M., Straub, C., Watanabe, M., and Tomita, S. (2013). Homeostatic Control of Synaptic Transmission by Distinct Glutamate Receptors. Neuron 78, 687-699.

Yang, Y.-M., Aitoubah, J., Lauer, A.M., Nuriya, M., Takamiya, K., Jia, Z., May, B.J., Huganir, R.L., and Wang, L.-Y. (2011). GluA4 is indispensable for driving fast neurotransmission across a high-fidelity central synapse. J. Physiol. 589, 42094227.

Zamanillo, D. (1999). Importance of AMPA Receptors for Hippocampal Synaptic Plasticity But Not for Spatial Learning. Science 284, 1805-1811.

Zhao, Y., Chen, S., Swensen, A.C., Qian, W.-J., and Gouaux, E. (2019). Architecture and subunit arrangement of native AMPA receptors elucidated by cryo-EM. Science 364, 355-362.

Zhu, J.J., Esteban, J.A., Hayashi, Y., and Malinow, R. (2000). Postnatal synaptic potentiation: delivery of GluR4-containing AMPA receptors by spontaneous activity. Nat. Neurosci. 3, 1098-1106. 